\title{
ON DIFFERENTIAL OPERATORS AND CONNECTIONS ${ }^{1}$ )
}

\author{
BY \\ H. K. NICKERSON
}

In this paper we construct, for an arbitrary principal bundle (with group a real Lie group) over a differentiable manifold, a sheaf of germs of linear differential operators of first order operating on the sheaf of germs of sections of any differentiable vector bundle associated with the given principal bundle. The commutators of these operators define a structure of Lie algebra (over the real numbers) on the operator sheaf. By considering the sheaves of germs of differential forms on the manifold with values in appropriate vector bundles, we obtain a graded sheaf of operators carrying a structure of graded Lie algebra (over the real numbers).

In many respects, the present work represents a generalization of the paper of Frölicher and Nijenhuis [2] which characterized the derivations (of all degrees) of the exterior algebra of real-valued differential forms on a differentiable manifold. The case considered by these authors is obtained by choosing the associated vector bundle to be the product bundle with fibre the real numbers. In addition, the usual theory of covariant differentiation, corresponding to a connection in the given principal bundle, appears as a special case.

It is hoped that the theory developed here will also have applications in the systematic study of deformation of the structures defined on a differentiable manifold by continuous pseudogroups of transformations.

1. Fundamental sequence of vector bundles. Let $M$ be a differentiable (i.e., $C^{\infty}$ ) manifold and let $P \rightarrow M$ be a differentiable principal bundle with group $G$, where $G$ is a real Lie group. Let $T(M), T(P)$, and $T(G)$ denote the bundle spaces of the tangent bundles to $M, P$, and $G$ respectively. Then $T(G)$ is also a Lie group, and $T(P) \rightarrow T(M)$ is a principal bundle with group $T(G)[3]$.

Since $G$ is a subgroup of $T(G)$, we may form the quotient bundle $T(P) / G$ $\rightarrow T(M)$, and we have

$$
0 \rightarrow K \stackrel{\iota}{\rightarrow} T(P) / G \stackrel{\pi}{\rightarrow} T(M) \rightarrow 0
$$

where $K$ denotes the restriction of $T(P) / G$ to $M \subset T(M)$. An element $\sigma$ of $T(P) / G$ over $u=\pi \sigma$, where $u$ is tangent to $M$ at $x$, represents an equivalence class of tangents to $P$ at points of the fibre $P_{x}$ over $x$; the members of the equivalence class are obtainable from any one member by right translation

Received by the editors January 4, 1961.

(1) This work was supported by a research grant from the National Science Foundation. 
by elements of $G$ and all tangents in the class project into $u$ under the map induced by the projection $P \rightarrow M$. In particular, an element of $K$ represents an equivalence class of vertical vectors, i.e., vectors along a fibre of $P$.

By a well-known theorem, $T(P) / G \rightarrow T(M)$ is isomorphic to the bundle over $T(M)$, (weakly) associated with $T(P) \rightarrow T(M)$, with group $T(G)$ and fibre $T(G) / G$.

The fibre $T(G) / G$ may be identified with $\mathfrak{g}$, where $\mathfrak{g}$ denotes the Lie algebra of $G$. In fact, the group $T(G)$ is isomorphic to a group constructed on the product space $G \times \mathrm{g}$. This construction may be made in two different ways, according as $g$ is identified with the Lie algebra of left-invariant or of right-invariant vector fields on $G$. We shall suppose that $\mathfrak{g}$ is identified with the Lie algebra of left-invariant vector fields on $G$. Then the multiplication in $G \times g$ is defined by

$$
(g, \lambda)(h, \mu)=\left(g h,\left(\operatorname{Ad~} h^{-1}\right) \lambda+\mu\right), \quad g, h \in G, \lambda, \mu \in g ;
$$

and $t \in T(G)$, tangent to $G$ at $g \in G$, corresponds to $\left(g, g^{-1} t\right) \in G \times g$. If $g$ is identified with the quotient space $T(G) / G$, where $G$ acts on $T(G)$ on the right, then $t=(g, \lambda) \in T(G)$ is projected into the equivalence class (Ad $g$ ) $\lambda$, and the induced left action of $T(G)$ on the quotient $g$ is expressed by

$$
t \mu=(\operatorname{Ad} g)(\lambda+\mu) \text {, }
$$

$$
t=(g, \lambda) \in T(G), \mu \in \mathfrak{g} .
$$

Let $\left\{U_{i}\right\}$ be a suitable open covering of $M$ and let

$$
g_{i k}: U_{i} \cap U_{k} \rightarrow G
$$

be the corresponding transition functions of the given principal bundle $P \rightarrow M$. Then

$$
\gamma_{i k}=g_{i k *}: T\left(U_{i}\right) \cap T\left(U_{k}\right) \rightarrow T(G)
$$

are transition functions for the principal bundle $T(P) \rightarrow T(M)$.

For the associated bundle with group $T(G)$ and fibre $\mathfrak{g}$, the transition laws are expressed by $\lambda_{i}=\gamma_{i k}(u) \lambda_{k}$, where $u \in T\left(U_{i}\right) \cap T\left(U_{k}\right)$ is tangent to $M$ at $x \in U_{i} \cap U_{k}$, and where $\left(u, \lambda_{i}\right) \in T\left(U_{i}\right) \times \mathfrak{g}$ and $\left(u, \lambda_{k}\right) \in \dot{T}\left(U_{k}\right) \times \mathfrak{g}$ correspond to the same bundle point. Explicitly, using (2), we have

$$
\lambda_{i}=\operatorname{Ad} g_{i k}(x) \lambda_{k}+\gamma_{i k}(u) g_{i k}^{-1}(x)
$$

where $\gamma_{i k}(u)=g_{i k *}(u)$. In particular, the bundle $K \rightarrow M(u=0)$ is seen to be isomorphic to the bundle over $M$, associated with $P \rightarrow M$, with group $G$ and fibre $\mathfrak{g}$, where $G$ acts on $\mathfrak{g}$ by the adjoint action.

The canonical isomorphism of the associated bundle $T(P) \times_{T(G)} \mathfrak{g} \rightarrow T(M)$ with the quotient bundle $T(P) / G \rightarrow T(M)$ will be used to introduce differentiable fibre coordinates in the latter bundle.

It is clear from (3) that $T(P) / G \rightarrow T(M)$ is not a vector bundle, but that 
$T(P) / G \rightarrow M$ is a vector bundle since $\gamma_{i k}$ is linear on $(T(M))_{x}, x \in U_{i} \cap U_{k}$. The bundles $K \rightarrow M$ and $T(M) \rightarrow M$ are also vector bundles, and it is easily verified that the sequence (1) represents an exact sequence of vector bundles over $M$, derived from the given principal bundle $P \rightarrow M[1]$.

2. Basic operation. The elementary notions which will be used below may be described as follows. Let $V$ and $W$ be differentiable manifolds, let $t$ be a tangent vector to $V$ at $v$, and let $\xi$ be a differentiable $W$-valued function defined on a differentiable curve through $v$ having tangent $t$ at $v$. These data determine, first of all, a curve in $V \times W$ through $(v, \xi(v))$ which "lies over" the given curve, that is, which projects into the given curve under the canonical projection $V \times W \rightarrow V$, and whose tangent $\eta$ at $(v, \xi(v))$ lies over $t$. If $\xi$ is given in a neighborhood of $v$, the vector $\eta$ is the tangent to the surface defined by $\xi$, lying over $t$. Since $T(V \times W)$ may be identified with $T(V) \times T(W)$, where $(T(V \times W))_{(v, w)}=(T(V))_{v} \oplus(T(W))_{w}$, we have a canonical decomposition

$$
\eta=t+\xi_{*}(t)
$$

where $\xi_{*}(t) \in(T(W))_{\xi(v)}$ is defined by considering $\xi$ as a mapping from (a subset of) $V$ into $W$. (If $\xi$ is considered as a mapping from (a subset of) $V$ into $V \times W$, then the symbol $\xi_{*}(t)$ would denote the vector $\eta$.) If we specialize to the case that $W$ is a finite dimensional vector space, then $(T(W))_{w}$ is isomorphic to $W$, and $\xi_{*}(t)$ may be identified with the Fréchet derivative $t \cdot \xi \in W$ of the $W$-valued function $\xi$ in the direction $t$. The Fréchet derivative $t \cdot \xi \in W$ may be computed in terms of ordinary differentiation of functions as follows: relative to a basis for $W$, the $W$-valued function $\xi$ is given by its components $\xi^{\mu}$; then $t \cdot \xi \in W$ has the components $t \cdot \xi^{\mu}$. Our basic operation will be induced by the Fréchet derivative $t \cdot \xi$; the fact that we have

$$
t \cdot \xi \sim \xi_{*}(t)=\eta-t
$$

leads to a geometric interpretation of this operation.

Let $B \rightarrow M$ be a bundle associated with the given principal bundle $P \rightarrow M$, with fibre $F$ and group $G$. Then $T(B) \rightarrow T(M)$ is a bundle associated with the principal bundle $T(P) \rightarrow T(M)$, with fibre $T(F)$ and group $T(G)$ [3]. We shall denote by

$$
\begin{aligned}
j: & P \times F & \rightarrow B=P \times_{G} F, \\
j_{*}: & T(P) \times T(F) & \rightarrow T(B)=T(P) \times_{T(G)} T(F)
\end{aligned}
$$

the projections used in defining the associated bundles. Then

$$
j(p g, f)=j(p, g f), \quad p \in P, f \in F, g \in G,
$$

and $b=j(p, f) \in \bigotimes_{x}$ if $p \in P_{x}, x \in M$. Similarly,

$$
j_{*}(t \gamma, z)=j_{*}(t, \gamma z), \quad t \in T(P), z \in T(F), \gamma \in T(G) .
$$

If $t$ is tangent to $P$ at $p$, and $z$ is tangent to $F$ at $f$, then $j_{*}(t, z)$ is tangent to 
$B$ at $j(p, f)$. Moreover, $t$ and $j_{*}(t, z)$ project into the same tangent vector in $T(M)$. Here we have identified $T(P \times F)$ with $T(P) \times T(F)$, where $(T(P \times F))_{(p, f)}$ is isomorphic to $(T(P))_{p} \oplus(T(F))_{f}$.

For given $b \in B_{x}$, the equation

$$
b=j(p, f),
$$

$$
p \in P, f \in F
$$

determines $f$ as an implicit function of $p$, for $p \in P_{x}$. The resulting $F$-valued function $\xi$ satisfies

$$
\xi(p g)=g^{-1} \xi(p), \quad g \in G,
$$

because of (7). A differentiable local section $s$ of $B \rightarrow M$ over an open set in $M$ determines a differentiable $F$-valued function $\xi$ on an open set in $P$ by allowing $b$ in (9) to vary over im $s$.

An element $\sigma$ of $T(P) / G$ in the fibre over $x \in M$ determines a unique tangent vector $\sigma_{p}$ to $P$ at each $p \in P_{x}$, with

$$
\sigma_{p} g=\sigma_{p g}, \quad g \in G,
$$

all vectors lying over $\pi \sigma$. If the fibre $F$ of $\beta \rightarrow M$ is a finite dimensional vector space, and if $\xi$ denotes the $F$-valued function determined by a (differentiable) local section $s$ of $B \rightarrow M$ defined in a neighborhood of $x$, then the Fréchet derivative $\sigma_{p} \cdot \xi \in F$ is defined for each $p \in P_{x}$. Moreover, if the action of $G$ on $F$ is linear, this $F$-valued function on $P_{x}$ has the property (10). In fact, for any fixed $g \in G$, we have

$$
\sigma_{p o} \cdot \xi=\sigma_{p} g \cdot \xi=\sigma_{p} \cdot(\xi \circ g)=\sigma_{p} \cdot g^{-1} \xi=g^{-1}\left(\sigma_{p} \cdot \xi\right) .
$$

In the first steps above, $g$ represents the map of $P_{x}$ into itself corresponding to right translation by $g$; we then use (10) to evaluate the composite map $\xi \circ g$, and finally the fact that the action of $g$ on $F$ is linear. Thus, if $\beta \rightarrow M$ is a vector bundle, we may set

$$
\sigma \cdot s=j\left(p, \sigma_{p} \cdot \xi\right), \quad p \in P_{x},
$$

where $\sigma_{p}$ is determined by $\sigma$, and $\xi$ by $s$. The value $\sigma \cdot s \in B_{x}$ defines the operation of $\sigma \in(T(P) / G)_{x}$ on the local section $s$ of the vector bundle $B \rightarrow M$, where $s$ is defined in an open neighborhood of $x, x \in M$.

A geometric interpretation may be obtained from (5), with $V=P, W=F$, $v=p \in P_{x}$, and $t=\sigma_{p}$. Here we map the right-hand member of (5) into $T(B)$ by $j_{*}$, rather than the left-hand member into $B$ by means of $j$. The vector $\eta \in T(P \times F)$, which is the tangent to $\mathrm{im} \xi$ lying over $\sigma_{p} \in T(P)$, maps into the tangent, lying over $\pi \sigma$, to im $s$ at $s(x)$; the vector $t=\left(\sigma_{p}, 0\right)$ maps into a vector which is tangent to $B$ at $j(p, f)=s(x)$, also lying over $\pi \sigma$. The difference $j_{*}\left(\xi_{*}\left(\sigma_{\xi}\right)\right)=j_{*}\left(\eta-\sigma_{p}\right)$ is therefore a vertical vector at $s(x)$, i.e., a tangent along the fibre $B_{x}$. These statements can be made without assuming that $B \rightarrow M$ is a vector bundle, but this assumption is required in order to map the vertical 
vector $j_{*}\left(\xi_{*}\left(\sigma_{p}\right)\right)$ canonically into an element of $B_{x}$ which, in fact, coincides with $\sigma \cdot s=j\left(p, \sigma_{p} \cdot \xi\right)$.

Next we show how the above operation may be computed in terms of local fibre coordinates. Recall that a coordinate function

$$
\phi: \quad U \times G \rightarrow P \mid U
$$

for the given principal bundle $P \rightarrow M$ induces a corresponding coordinate function

$$
\hat{\phi}: \quad U \times F \rightarrow B \mid U
$$

by setting

$$
\hat{\phi}(x, f)=j(\phi(x, e), f), \quad x \in U, f \in F,
$$

where $e$ denotes the identity element of $G$; that is, $b=j(p, f)$ is assigned the coordinates $(x, f)$ if $p$ has coordinates $(x, e)$. A local section $s$ of $\beta \rightarrow M$ over $U$ is determined by the fibre coordinate $f(x)$ of $s(x)$, as $x$ varies. From (10), we then see that the $F$-valued function $\xi$ on $P \mid U$ is given by

$$
\xi(p)=g^{-1} f(x) \quad \text { for } p=\phi(x, g), g \in G .
$$

From

$$
\phi_{*}: \quad T(U) \times T(G) \rightarrow T(P) \mid T(U)
$$

and

$$
T(P) / G \approx T(P) \times_{T(G)} \mathfrak{g},
$$

we find that $\sigma \in(T(P) / G)_{x}$ has coordinates $(u, \lambda)$, with $u=\pi \sigma \in(T(M))_{x}$ and $\lambda \in g$, if

$$
\phi_{*}(u, \lambda)=\sigma_{p} \quad \text { for } p=\phi(x, e) .
$$

Here $g$ is identified with $(T(G))_{e}$. Computing at $p=\phi(x, e)$, we then have

$$
\sigma_{p} \cdot \xi=u \cdot f-\lambda(f),
$$

where $\lambda(f)$ denotes the action of $\lambda \in \mathfrak{g}$ on $f \in F$ under the representation of $g$ in $F$ induced by the linear action of $G$ on $F$. The minus sign occurs because $g^{-1}$, rather than $g$, appears on the right-hand side of (13). The right-hand side of (14) then gives the fibre coordinate of $\sigma \cdot s \in B_{x}$.

Still more explicitly, suppose that $G \subset G L(m, R)$, where $R$ denotes the real numbers. Then $\lambda \in g$ is an $(m \times m)$-matrix $\left(\lambda_{\nu}^{\mu}\right), \mu, \nu=1, \cdots, m$. If the fibre $F$ of $B \rightarrow M$ is $R^{m}$, then the local section $s$ of $B \rightarrow M$ is represented by functions $f^{\mu}(x), \mu=1, \cdots, m$, and $\sigma \cdot s \in B_{x}$ is represented by

$$
(\sigma \cdot s)^{\mu}=u \cdot f^{\mu}-\lambda_{v}^{\mu} f^{\prime}, \quad \mu=1, \cdots, m .
$$


If $B \rightarrow M$ is replaced by its dual bundle with fibre $\left(R^{m}\right)^{*}$, a local section $s$ being represented by fibre coordinates $h_{\nu}, \nu=1, \cdots, m$, then $\sigma \cdot s$ is represented by

$$
(\sigma \cdot s)_{\nu}=u \cdot h_{\nu}+h_{\mu} \lambda_{\nu}{ }_{\nu}, \quad \nu=1, \cdots, m .
$$

For more general choices of the fibre $F$ of $B \rightarrow M$, the pattern of the terms representing the action of the matrix $\left(\lambda_{\nu}^{\mu}\right)$ on the coordinates of $s$ is determined by the appropriate analogue of the expression of the action of $\mathfrak{g} C \mathrm{gl}(m, \boldsymbol{R})$ on the vector space $F$. For example, if the fibre of $\beta \rightarrow M$ is $R^{m} \otimes R^{m}$, with $s$ represented by functions $f^{\mu \rho}$, then $\sigma \cdot s$ is represented by

$$
(\sigma \cdot s)^{\mu \rho}=u \cdot f^{\mu \rho}-\lambda_{\nu}^{\mu} f^{\nu \rho}-\lambda_{\nu}^{\rho} f^{\mu \nu}, \quad \mu, \rho=1, \cdots, m .
$$

For given $\sigma \in(T(P) / G)_{x}$, we may compute the value of $\sigma \cdot s \in \bigotimes_{x}$ if the values of $s$ are given only on a curve through $x$ having the tangent $\pi \sigma$ at $x$. In particular, for $\sigma \in K \subset T(P) / G$ (i.e., $\pi \sigma=0$ ), the value $\sigma \cdot s \in \bigotimes_{x}$ is defined for any $\sigma \in K_{x}$ and $s \in \bigotimes_{x}$. However, in order that $\sigma \cdot s$ be defined for all choices of $\sigma \in(T(P) / G)_{x}$, it is necessary that $s$ be given as a differentiable local section of $B \rightarrow M$ on an open neighborhood of $x$. This action then maps local sections into bundle points. From the representation (14) in terms of fibre coordinates, it is clear that the action of $\sigma$ varies differentiably with $\sigma$. So, if $\sigma$ is given as a differentiable local section of $T(P) / G \rightarrow M$, defined on the same open set of $M$ as $s$, the values $\sigma \cdot s$ determine a local section of $B \rightarrow M$. Furthermore, it is easily seen from (12) that the operation of $\sigma$ on $s$ is "local" in the sense that $\sigma=\hat{\sigma}$ on an open set $U \subset M$, on which $s$ is also defined, implies $\sigma \cdot s=\hat{\sigma} \cdot s$ in $U$, and that $s=\hat{s}$ in $U$ implies $\sigma \cdot s=\sigma \cdot \hat{s}$ in $U$. Thus the operation (12) leads to a well-defined operation, at each $x \in M$, for elements in the sheaves of germs of differentiable sections of the respective vector bundles. It is also clear from (12) that this operation is $R$-linear.

3. Sheaves of operators. Let $\Xi^{q}, \Sigma^{q}, B^{q}$, and $S^{q}$ denote the sheaves of germs of (differentiable) differential forms of degree $q$ on $M$ with values in the vector bundles $K \rightarrow M, T(P) / G \rightarrow M, T(M) \rightarrow M$, and $B \rightarrow M$ respectively, where $B \rightarrow M$ is an arbitrary vector bundle associated with the given principal bundle $P \rightarrow M$. The sheaf $S^{q}$ depends on the choice of the bundle $B \rightarrow M$. The germs of forms of degree 0 correspond to the germs of differentiable sections of the appropriate vector bundles. The elements of $B^{q}$ are the germs of vector-valued $q$-forms on $M$. We shall denote by $A^{q}$ the sheaf of germs of (differentiable) real-valued differential forms of degree $q$ on $M$, or $q$-forms with values in the product bundle $M \times R \rightarrow M$. Then $A^{0}$ is the sheaf of germs of differentiable functions on $M$, and the above sheaves are sheaves of $A^{0}$. modules. Obviously, $\Xi^{q} \subset \Sigma^{a}$ and each element $\sigma$ in $\Sigma^{q}$ determines an element of $B^{q}$ which we shall denote by $\pi \sigma$, with $\sigma \in \Xi^{q}$ if and only if $\pi \sigma=0$. Thus we have the exact sequences 


$$
0 \rightarrow \Xi^{q} \stackrel{\iota}{\rightarrow} \Sigma^{q} \stackrel{\pi}{\rightarrow} B^{q} \rightarrow 0, \quad q=0,1, \cdots, n=\operatorname{dim} M,
$$

of sheaves of $A^{0}$-modules, the case $q=0$ corresponding to the sheaves of germs of differentiable sections of the sequence (1).

By (12), we have defined $\Sigma^{0}$ as an operator sheaf for the sheaf $S^{0}$ of germs of differentiable sections of any vector bundle $B \rightarrow M$ associated with $P \rightarrow M$. We wish to extend the action (12) to define a map

$$
\Sigma^{q} \otimes_{R} S^{p} \rightarrow S^{q+p}
$$

sending $\sigma \otimes \Phi$, where $\sigma \in \Sigma_{x}^{q}, \Phi \in S_{x}^{p}$, into $\sigma \cdot \Phi \in S_{x}^{q+p}$.

It will henceforth be assumed-without explicit mention-that all germs in any operation or computation are elements in the stalks, of the various sheaves, over a common point $x \in M$. All formulas may, of course, also be interpreted as formulas for representatives (denoted by the same symbols) of the relevant germs on a common open neighborhood of the given point $x \in M$. If $v \in B^{0}$ and if $t$ is the germ of a differentiable section of a tensor bundle on $M$, we shall denote by $v \square t$ the germ which corresponds to the classical Lie derivative of a representative of $t$ with respect to a representative of $v$. We shall denote by $U_{r}$ a generic element of $\Lambda^{r} B^{0}$ corresponding to $u_{1} \wedge \cdots \wedge u_{r}, u_{i} \in B^{0}, i=1, \cdots, r$. We shall write $U_{I}$ for $u_{i_{1}} \wedge \cdots \wedge u_{i q}$, where $I=I_{q}=\left(i_{1}<\cdots<i_{q}\right), q>0$, with $U_{I}=1$ if $q=0$ and $U_{I}=0$ if $q<0$. For $K=K_{2}$, we shall write $\left[U_{K}\right]$ for the Poisson bracket $\left[u_{k_{1}}, u_{k_{2}}\right]=u_{k_{1}} \square u_{k_{2}}$. The symbol $\epsilon_{I J}$ will denote the sign of the permutation $i_{1} \cdots i_{q} j_{1} \cdots j_{p}$ of the integers $1,2, \cdots, q+p$, or zero in the case that $I=I_{q}$ and $J=J_{p}$ have an integer in common. Finally, if $\sigma \in \Sigma^{q}$, for example, the symbol $\left\langle U_{I}, \sigma\right\rangle \in \Sigma^{\bullet}$ will denote the value of the $q$-form $\sigma$ for the argument $U_{I}$, where $I=I_{q}$.

Let $\sigma \in \Sigma^{q}, \Phi \in S^{p}$. Then $\sigma \cdot \Phi \in S^{q+p}$ is defined by the values $\left({ }^{2}\right)$

$$
\begin{aligned}
\left\langle U_{q+p}, \sigma \cdot \Phi\right\rangle= & \sum_{I_{q}, J_{p}} \epsilon_{I J}\left\{\left\langle U_{I}, \sigma\right\rangle \cdot\left\langle U_{J}, \Phi\right\rangle-\left\langle\left\langle U_{I}, \pi \sigma\right\rangle \square U_{J}, \Phi\right\rangle\right\} \\
& +(-1)^{q-1} \sum_{K_{2}, L_{q-1}, M_{p-1}} \epsilon_{K L M}\left\langle\left\langle\left[U_{K}\right] \wedge U_{L}, \pi \sigma\right\rangle \wedge U_{M}, \Phi\right\rangle .
\end{aligned}
$$

The essential action of $\sigma$ is expressed by the operation of $\Sigma^{0}$ on $S^{0}$ in the terms $\left\langle U_{I}, \sigma\right\rangle \cdot\left\langle U_{J}, \Phi\right\rangle$; the remaining terms, involving Lie derivatives, are needed to ensure that $\sigma \cdot \Phi$ define an $A^{0}$-linear map from $\Lambda^{q+p} B^{0}$ into $S^{0}$, that is, that $\sigma \cdot \Phi$ be a germ of a differential form of degree $q+p$ on $M$ with values in the vector bundle $B \rightarrow M$, or $\sigma \cdot \Phi \in S^{q+p}$. The operation defined by (20) is clearly $\boldsymbol{R}$-linear (so as to give (19)) but is not, in general, $A^{0}$-linear. However, for $\sigma \in \Xi^{q} \subset \Sigma^{q}$, that is, $\pi \sigma=0$, the action of $\sigma$ is $A^{0}$-linear, or

$$
\Xi^{q} \otimes A^{0} S^{p} \rightarrow S^{q+p} \text {. }
$$

(2) Added in proof: It has been pointed out by C. J. Henrich that the same result can be obtained by generalizing (12), replacing ordinary differentiation of component functions by the (generalized) Lie derivation of forms. 
If $G \subset G L(m, R)$, there are no trivial operators in $\Sigma^{q}$; that is, $\sigma \cdot \Phi=0$ for all $\Phi \in S^{p}, 0 \leqq p \leqq n$, for all choices of the associated vector bundle $B \rightarrow M$, implies $\sigma=0$. In fact, it is sufficient to assume $\sigma \cdot \Phi=0$ for all $\Phi \in S^{0}$ where $B \rightarrow M$ is the vector bundle with fibre $F=\boldsymbol{R}^{m}$. For $\sigma \in \Sigma^{0}$, the conclusion then follows easily from the local representation (15) and, for $\sigma \in \Sigma^{q}$, from the fact that the formula (20) reduces, for $p=0$, to $\left\langle U_{q}, \sigma \cdot \Phi\right\rangle=\left\langle U_{q}, \sigma\right\rangle \cdot \Phi$ where $\left\langle U_{q}, \sigma\right\rangle$ $\in \Sigma^{0}$

If $P \rightarrow M$ is the trivial principal bundle, with $G=e, \mathfrak{g}=0$, then $\Xi q=0$, $\Sigma^{q} \approx B^{q}$, and $S^{p}=A^{p}$ if we take $B \rightarrow M$ to be the product bundle $M \times R \rightarrow M$. We shall show below that, in this case $\left(\sigma=\pi \sigma, \Phi=\phi \in A^{p}\right)$, formula (20) gives

$$
\pi \sigma \cdot \phi=d_{\pi \sigma} \phi, \quad \pi \sigma \in B^{q}, \phi \in A^{p} ;
$$

here the right-hand member denotes the (generalized) Lie derivative of the $p$-form $\phi$ with respect to the vector-valued $q$-form $\pi \sigma$, in the notation of Frôlicher and Nijenhuis [2].

We can also obtain local representations of (20) corresponding to the case of an arbitrary principal bundle $P \rightarrow M$ with group $G \subset G L(m, R)$. Then $\sigma \in \Sigma^{q}$ is represented locally by a pair consisting of $\pi \sigma \in B^{q}$ and an $(m \times m)$ matrix $\left(\sigma_{\nu}^{\mu}\right)$ of (germs of) $q$-forms. Although (20) is valid for any choice of the associated vector bundle $B \rightarrow M$, a local representation of $\Phi \in S^{p}$ can be given only for each particular choice of $B \rightarrow M$. If $B \rightarrow M$ has fibre $F=R^{m}$, then $\Phi \in S^{p}$ is represented by $p$-forms $\phi^{\mu}, \mu=1, \cdots, m$. We shall show that $\sigma \cdot \Phi \in S^{q+p}$ is represented by the $(q+p)$-forms

$$
(\sigma \cdot \Phi)^{\mu}=d_{\pi \sigma} \phi^{\mu}-\sigma_{\nu}^{\mu} \wedge \phi^{\prime}, \quad \quad \mu=1, \cdots, m .
$$

This formula includes (15) as a special case, by taking $q=p=0$. For other choices of $B \rightarrow M$, generalizations of (16), (17), etc., are obtained. In particular, if we choose $B \rightarrow M$ to have fibre $F=R$ where $G$ acts trivially on $F$, then $S^{p}=A^{p}$ and

$$
\sigma \cdot \phi=d_{\pi \sigma} \phi, \quad \sigma \in \Sigma^{q}, \phi \in A^{p} .
$$

Clearly, $\sigma \cdot \phi=0$ for $\sigma \in \Xi^{q}$.

To express the generalized Lie derivative for forms, Frölicher and Nijenhuis used a contraction operation of real-valued (or vector-valued) forms with a vector-valued form. We summarize here the definition and properties of this operation in a more general setting.

Let $X^{p}$ denote the sheaf of germs of $p$-forms on $M$ with values in some vector bundle over $M$ (for example, $X^{p}=S^{p}$, or $\Sigma^{p}$, or $B^{p}$ or $A^{p}$ ). For $\Phi \in X^{p}$ and $V \in B^{q}$, the contraction $\Phi \pi V \in X^{p+q-1}$ is defined by the values

$$
\left\langle U_{p+q-1}, \Phi \pi V\right\rangle=\sum_{I q, M_{p-1}} \epsilon_{I M}\left\langle\left\langle U_{I}, V\right\rangle \wedge U_{M} . \Phi\right\rangle .
$$

This operation is clearly $A^{0}$-linear and so induces a map 


$$
X^{p} \dot{\otimes}_{A^{0}} B^{q} \rightarrow X^{p+q-1} .
$$

We have $\Phi \pi V=0$ if $p=0$, since then $U_{M}=0$; for $p=1$ and $u \in B^{0}$, we have $\Phi \pi u=\langle u, \Phi\rangle$. If $\mu$ is an $A^{0}$-homomorphism of the range $X^{0}$ of $\Phi$ into another sheaf over $M$, then

$$
\mu(\Phi \pi V)=(\mu \Phi) \pi V .
$$

For $V \in B^{q}, W \in B^{r}$ and $\Phi \in X^{p}$, we have the identity

(26) $\Phi \pi(V \pi W)-(\Phi \pi V) \pi W=(-1)^{(q-1)(r-1)}\{\Phi \pi(W \pi V)-(\Phi \pi W) \pi V\}$

with both sides of (26) vanishing if $p=1$. Finally, if $\delta \in H^{0}\left(M, B^{1}\right)$ is the canonical 1 -form corresponding to the identity transformation of $B^{0}$ into itself, that is,

$$
\langle u, \delta\rangle=u, \quad u \in B^{0},
$$

then

$$
\Phi \pi \delta=p \Phi, \quad \delta \pi V=V, \quad \Phi \in X^{p}, V \in B^{q} .
$$

In terms of the contraction operation, the (generalized) Lie derivative for forms is given by

$$
d_{V} \phi=d \phi \pi V+(-1)^{q} d(\phi \pi V), \quad V \in B^{q}, \phi \in A^{p},
$$

where $d=d_{\delta}$ is the exterior derivative. This obviously reduces to the standard formula

$$
v \square \phi=d \phi \pi v+d(\phi \pi v), \quad v \in B^{0}, \phi \in A^{p},
$$

when $q=0, V=v$.

In the case that the given principal bundle is trivial, we have $\Sigma q \approx B^{q}$ and the "basic action" of $\S 2$ is ordinary differentiation, by (14). For $\phi \in A^{p}$ and $\sigma=\pi \sigma \in B^{q}$, the formula (20) becomes

$$
\begin{aligned}
\left\langle U_{q+p}, \pi \sigma \cdot \phi\right\rangle= & \sum_{I_{q}, J_{p}} \epsilon_{I J}\left\{\left\langle U_{I}, \pi \sigma\right\rangle \cdot\left\langle U_{J}, \phi\right\rangle-\left\langle\left\langle U_{I}, \pi \sigma\right\rangle \square U_{J}, \phi\right\rangle\right\} \\
& +(-1)^{q-1} \sum_{K_{2}, L_{q-1}, M_{p-1}} \epsilon_{K L M}\left\langle\left\langle\left[U_{K}\right] \wedge U_{L}, \pi \sigma\right\rangle \wedge U_{M}, \phi\right\rangle .
\end{aligned}
$$

For $q=0, \pi \sigma=v \in B^{0}$, this reduces to another well-known formula

$$
\left\langle U_{p}, v \square \phi\right\rangle=v \cdot\left\langle U_{p}, \phi\right\rangle-\left\langle v \square U_{p}, \phi\right\rangle, \quad v \in B^{0}, \phi \in A^{p} .
$$

To show that the two generalizations (29) and (31) define the same operation, for $V=\pi \sigma$, we first combine (30) and (32) to give, for $v \in B^{0}, \phi \in A^{p}$,

$$
\begin{aligned}
\left\langle v \wedge U_{p}, d \phi\right\rangle & =\left\langle U_{p}, d \phi \pi v\right\rangle=\left\langle U_{p}, v \square \phi\right\rangle-\left\langle U_{p}, d(\phi \pi v)\right\rangle \\
& =v \cdot\left\langle U_{p}, \phi\right\rangle-\left\langle v \square U_{p}, \phi\right\rangle-\left\langle U_{p}, d(\phi \pi v)\right\rangle .
\end{aligned}
$$


Then, for $V \in B^{q}, \phi \in A^{p}$, we have

$$
\begin{aligned}
\left\langle U_{q+p}, d \phi \pi V\right\rangle & =\sum_{I_{q}, J_{p}} \epsilon_{I J}\left\langle\left\langle U_{I}, V\right\rangle \wedge U_{J}, d \phi\right\rangle \\
= & \sum_{I_{q}, J_{p}} \epsilon_{I J}\left\{\left\langle U_{I}, V\right\rangle \cdot\left\langle U_{J}, \phi\right\rangle-\left\langle\left\langle U_{I}, V\right\rangle \square U_{J}, \phi\right\rangle\right. \\
& \left.-\left\langle U_{J}, d\left(\phi \pi\left\langle U_{I}, V\right\rangle\right)\right\rangle\right\} .
\end{aligned}
$$

From the formula

$$
\left\langle U_{p+1}, d \psi\right\rangle=\sum_{i, J_{p}} \epsilon_{i J} u_{i} \cdot\left\langle U_{J}, \psi\right\rangle-\sum_{K_{2}, M_{p-1}} \epsilon_{K M}\left\langle\left[U_{K}\right] \wedge U_{M}, \psi\right\rangle, \psi \in A^{p},
$$

for the exterior derivative, we compute

$$
\begin{aligned}
\langle & \left.U_{q+p},(-1)^{q} d(\phi \pi V)\right\rangle \\
= & \sum_{s, S_{q+p-1}}(-1)^{q} \epsilon_{s} S u_{s} \cdot\left\langle U_{S}, \phi \pi V\right\rangle+(-1)^{q-1} \sum_{K_{2}, R_{q+p-2}} \epsilon_{K R}\left\langle\left\lfloor U_{K}\right] \wedge U_{R}, \phi \pi V\right\rangle \\
= & (-1)^{q} \sum_{s, I_{q}, M_{p-1}} \epsilon_{s I M} u_{s} \cdot\left\langle\left\langle U_{I}, V\right\rangle \wedge U_{M}, \phi\right\rangle \\
(35) \quad & +(-1)^{q-1}\left\{\sum_{K_{2}, L_{q-1}, M_{p-1}} \epsilon_{K L M}\left\langle\left\langle\left[U_{K}\right] \wedge U_{L}, V\right\rangle \wedge U_{M}, \phi\right\rangle\right. \\
& \left.\quad+(-1)^{q} \sum_{K_{2}, I_{q}, T_{p-2}} \epsilon_{K I T}\left\langle\left\langle U_{I}, V\right\rangle \wedge\left[U_{K}\right] \wedge U_{T}, \phi\right\rangle\right\} \\
= & \sum_{I_{q}, J_{p}} \epsilon_{I J}\left\langle U_{J}, d\left(\phi \pi\left\langle U_{I}, V\right\rangle\right)\right\rangle \\
& +(-1)^{q-1} \sum_{K_{2}, L_{q-1}, M_{p-1}} \epsilon_{K L M}\left\langle\left\langle\left[U_{K}\right] \wedge U_{L}, V\right\rangle \wedge U_{M}, \phi\right\rangle,
\end{aligned}
$$

using (-1) ${ }^{q} \epsilon_{s I}=\epsilon_{I s}$, etc. The sum of (33) and (35) gives $\left\langle U_{q+p}, d_{V} \phi\right\rangle$, by (29), and coincides with $\left\langle U_{q+p}, \pi \sigma \cdot \phi\right\rangle$, as given by (31), if $V=\pi \sigma$; this implies (22).

To prove (23), we use (15) to evaluate the components

$$
\left(\epsilon_{I J}\left\langle U_{I}, \sigma\right\rangle \cdot\left\langle U_{J}, \Phi\right\rangle\right)^{\mu}=\epsilon_{I J}\left\langle U_{I}, \pi \sigma\right\rangle \cdot\left\langle U_{J}, \phi^{\mu}\right\rangle-\epsilon_{I J}\left\langle U_{I},{ }^{\mu}{ }_{\nu}\right\rangle\left\langle U_{J}, \phi^{\nu}\right\rangle
$$

of the first expression on the right of (20). The terms of the first kind on the right of (36) combine with the corresponding components of the remaining terms on the right of $(20)$ to give $\left\langle U_{q+p}, d_{\pi \sigma} \phi^{\mu}\right\rangle$. The fact that the sum of the terms of the second kind on the right of (36) is $\left\langle U_{q+p},-\sigma_{\nu}^{\mu} \wedge \phi^{\nu}\right\rangle$ follows from

$$
\left\langle U_{q+p}, \psi \wedge \phi\right\rangle=\sum_{I_{q}, J_{p}} \epsilon_{I J}\left\langle U_{I}, \psi\right\rangle\left\langle U_{J}, \phi\right\rangle, \quad \psi \in A^{q}, \phi \in A^{p} .
$$

4. Graded Lie algebra. In this section we shall show that the graded sheaf $\Sigma^{*}=\left(\Sigma^{0}, \Sigma^{1}, \cdots, \Sigma^{n}, 0, \cdots\right)$ carries a structure of graded Lie algebra (over $\boldsymbol{R}$ ). That is, we shall define a map 


$$
\Sigma^{q} \otimes R \Sigma^{r} \rightarrow \Sigma^{q+r}
$$

sending $\sigma \otimes \tau$, where $\sigma \in \Sigma^{q}, \tau \in \Sigma^{r}$, into the bracket product $[\sigma, \tau] \in \Sigma^{q+r}$, where $[\sigma, \tau]$ satisfies

$$
[\sigma, \tau] \cdot \Phi=\sigma \cdot(\tau \cdot \Phi)-(-1)^{q r} \tau \cdot(\sigma \cdot \Phi),
$$

for all $\Phi \in S^{*}$, for all choices of the associated vector bundle $B \rightarrow M$ determining $S^{*}$, and

$$
\begin{aligned}
{[\sigma, \tau] } & =(-1)^{q r+1}[\tau, \sigma], \\
{[[\sigma, \tau], \omega] } & =[\sigma,[\tau, \omega]]-(-1)^{q r}[\tau,[\sigma, \omega]],
\end{aligned}
$$

for $\sigma \in \Sigma^{q}, \tau \in \Sigma^{r}, \omega \in \Sigma^{*}$. Formulas (41) and (40) together imply the Jacobi identity.

If $G \subset G L(m, R)$, the element $[\sigma, \tau] \in \Sigma^{q+r}$ is determined uniquely by the condition (39) and is therefore the commutator of the operators $\sigma$ and $\tau$; the condition (39) then implies that the map $(\sigma, \tau) \rightarrow[\sigma, \tau]$ is $R$-linear (even $A^{0}$ linear for $\left.\sigma, \tau \in \Xi^{*} \subset \Sigma^{*}\right)$ and that (40) and (41) are satisfied. In the general case it is necessary to check these properties from the formula (45) below which defines $[\sigma, \tau]$ in terms of $\sigma$ and $\tau$. For $R$-linearity (or $A^{0}$-linearity in $\left.\Xi^{*} \subset \Sigma^{*}\right)$ and (40), this is trivial; the details of the verification of (41) will be omitted.

Two germs $\sigma, \tau \in \Sigma^{0}$ represent right-invariant vector fields on $P$; the Poisson bracket of these vector fields is again a right-invariant vector field on $P$ and determines an element of $\Sigma^{0}$ which will be denoted by $[\sigma, \tau]$. Clearly we have

$$
\pi[\sigma, \tau]=[\pi \sigma, \pi \tau]
$$

where the right-hand side is defined by the Poisson bracket in $B^{0}$. The fact that this bracket satisfies (39) for $\Phi \in S^{0}$ follows immediately from the definition (12) since the Poisson bracket gives the commutator for differentiation of functions. For $\Phi \in S^{p}$ we have, by (20),

$$
\begin{aligned}
& \left\langle U_{p}, \sigma \cdot(\tau \cdot \Phi)-\tau \cdot(\sigma \cdot \Phi)\right\rangle \\
& =\sigma \cdot\left\{\tau \cdot\left\langle U_{p}, \Phi\right\rangle-\left\langle\pi \tau \square U_{p}, \Phi\right\rangle\right\}-\tau \cdot\left\langle\pi \sigma \square U_{p}, \Phi\right\rangle+\left\langle\pi \tau \square\left(\pi \sigma \square U_{p}\right), \Phi\right\rangle \\
& \quad-\tau \cdot\left\{\sigma \cdot\left\langle U_{p}, \Phi\right\rangle-\left\langle\pi \sigma \square U_{p}, \Phi\right\rangle\right\}+\sigma \cdot\left\langle\pi \tau \square U_{p}, \Phi\right\rangle-\left\langle\pi \sigma \square\left(\pi \tau \square U_{p}\right), \Phi\right\rangle \\
& =[\sigma, \tau] \cdot\left\langle U_{p}, \Phi\right\rangle-\left\langle[\pi \sigma, \pi \tau] \square U_{p}, \Phi\right\rangle=\left\langle U_{p},[\sigma, \tau] \cdot \Phi\right\rangle
\end{aligned}
$$

since $\left\langle U_{p}, \Phi\right\rangle \in S^{0}$, and the Poisson bracket also gives the commutator for classical Lie derivatives.

Thus, $\Sigma^{0}$ is a Lie algebra (over $R$ ). From (42) we see that $\Xi^{0}$ is an ideal in $\Sigma^{0}$ and that the bracket in $\Sigma^{0}$ projects into the Poisson bracket in $B^{0}$. In particular, the sequence (18) for $q=0$ is an exact sequence of sheaves of Lie algebras (over $\boldsymbol{R})$. 
An explicit computation shows that, if $\sigma$ and $\tau$ are represented locally by $(u, \lambda)$ and $(v, \eta)$ where $u=\pi \sigma, v=\pi \tau$ are vector fields on $M$ and $\lambda, \eta$ are $g$ valued functions, then $[\sigma, \tau]$ is represented by

$$
[(u, \lambda),(v, \eta)]=([u, v],[\lambda, \eta]+u \cdot \eta-v \cdot \lambda),
$$

where $[\lambda, \eta]$ denotes the Lie algebra bracket of $\lambda$ and $\eta$, and where the $g$ valued functions $u \cdot \eta$ and $v \cdot \lambda$ are Fréchet derivatives. In particular, we see that the bracket product in $\Xi^{0}$ is induced by the Lie algebra bracket in the fibre $\mathfrak{g}$ of the vector bundle $K \rightarrow M$, and is $A^{0}$-linear.

For $\sigma \in \Sigma^{q}, \tau \in \Sigma^{r}$, the element $[\sigma, \tau]$ in $\Sigma^{q+r}$ is defined, in terms of the bracket product in $\Sigma^{0}$, by the values

$$
\begin{aligned}
& \left\langle U_{q+r},[\sigma, \tau]\right\rangle \\
& =\sum_{I_{q}, J_{r}} \epsilon_{I J}\left\{\left[\left\langle U_{I}, \sigma\right\rangle,\left\langle U_{J}, \tau\right\rangle\right]-\left\langle\left\langle U_{I}, \pi \sigma\right\rangle \square U_{J}, \tau\right\rangle+\left\langle\left\langle U_{J}, \pi \tau\right\rangle \square U_{I}, \sigma\right\rangle\right\} \\
& \quad+(-1)^{q-1} \sum_{K_{2}, L_{q-1}, M_{r-1}} \epsilon_{K L M}\left\{\left\langle\left\langle\left[U_{K}\right] \wedge U_{L}, \pi \sigma\right\rangle \wedge U_{M}, \tau\right\rangle\right. \\
& \left.-\left\langle\left\langle\left[U_{K}\right] \wedge U_{M}, \pi \tau\right\rangle \wedge U_{L}, \sigma\right\rangle\right\} .
\end{aligned}
$$

It can then be verified, by a computation generalizing (43), that (45) implies (39), as required.

If in (39) we take the associated vector bundle to be the trivial bundle with fibre $R$, with $\Phi=\phi \in A^{*}$, we have by (24)

$$
\begin{aligned}
d_{\pi[\sigma, \tau]} \phi & =d_{\pi \sigma}\left(d_{\pi \tau} \phi\right)-(-1)^{q r} d_{\pi \tau}\left(d_{\pi \sigma} \phi\right) \\
& =d_{[\pi \sigma, \pi \tau]} \phi
\end{aligned}
$$

where $[\pi \sigma, \pi \tau]$ denotes the generalized Poisson bracket [2] for elements of $B^{*}$, which gives the commutator for the (generalized) Lie derivations. Thus (42) holds for arbitrary $\sigma, \tau \in \Sigma^{*}$, where now the right-hand side is defined by the generalized Poisson bracket in $B^{*}$.

Thus, the graded sheaf $\Xi^{*}$ is a graded ideal in $\Sigma^{*}$, and

$$
0 \rightarrow \Xi^{*} \stackrel{\iota}{\rightarrow} \Sigma^{*} \stackrel{\pi}{\rightarrow} B^{*} \rightarrow 0
$$

is an exact sequence of sheaves of graded Lie algebras (over $\boldsymbol{R})$. Moreover, the bracket product induced in $B^{*}$ is independent of the choice of the principal bundle $P \rightarrow M$ and coincides with the generalized Poisson bracket given by Frölicher and Nijenhuis.

If $\sigma \in \Sigma^{q}$ is represented locally by $\pi \sigma \in B^{q}$ and an $(m \times m)$-matrix $\left(\sigma_{\nu}^{\mu}\right)$ of $q$-forms, and $\tau \in \Sigma^{r}$ is represented by $\pi \tau \in B^{r}$ and an $(m \times m)$-matrix $\left(\tau_{\nu}^{\mu}\right)$ of $r$-forms, then $[\sigma, \tau] \in \Sigma^{q+r}$ is represented by $\pi[\sigma, \tau]=[\pi \sigma, \pi \tau] \in B^{q+r}$ and an $(m \times m)$-matrix $\left([\sigma, \tau]_{\nu}^{\mu}\right)$ of $(q+r)$-forms where

$$
[\sigma, \tau]_{\nu}^{\mu}=(-1)^{q r} \tau_{\rho}^{\mu} \wedge \sigma_{\nu}^{\rho}-\sigma_{\rho}^{\mu} \wedge \tau_{\nu}^{\rho}+d_{\pi \sigma} \tau_{\nu}^{\mu}-(-1)^{q r} d_{\pi \tau} \sigma_{\nu}^{\mu}
$$


In fact, if we take components in (45), evaluating $\left[\left\langle U_{I}, \sigma\right\rangle,\left\langle U_{J}, \tau\right\rangle\right]$ by means of (44), we find

$$
\begin{gathered}
\left\langle U_{q+r},[\sigma, \tau]_{\nu}^{\mu}\right\rangle=\sum_{I q, J_{r}} \epsilon_{I J}\left\{\left\langle U_{J}, \tau_{\rho}^{\mu}\right\rangle\left\langle U_{I}, \sigma_{\nu}^{\rho}\right\rangle-\left\langle U_{I}, \sigma_{\rho}^{\mu}\right\rangle\left\langle U_{J}, \tau_{\nu}^{p}\right\rangle\right. \\
+\left\langle U_{I}, \pi \sigma\right\rangle \cdot\left\langle U_{J}, \tau_{\nu}^{\mu}\right\rangle-\left\langle U_{J}, \pi \tau\right\rangle \cdot\left\langle U_{I}, \sigma_{\nu}^{\mu}\right\rangle \\
\left.\quad-\left\langle\left\langle U_{I}, \pi \sigma\right\rangle \square U_{J}, \tau_{\nu}^{\mu}\right\rangle+\left\langle\left\langle U_{J}, \pi \tau\right\rangle \square U_{I}, \sigma_{\nu}^{\mu}\right\rangle\right\} \\
+(-1)^{q-1} \sum_{K_{2}, L_{q-1}, M_{r-1}} \epsilon_{K L M}\left\{\left\langle\left\langle\left[U_{K}\right] \wedge U_{L}, \pi \sigma\right\rangle \wedge U_{M}, \tau_{\nu}^{\mu}\right\rangle\right. \\
\left.-\left\langle\left\langle\left[U_{K}\right] \wedge U_{M}, \pi \tau\right\rangle \wedge U_{L}, \sigma_{\nu}^{\mu}\right\rangle\right\}
\end{gathered}
$$

and the formula (47) then follows by (37), (31) and (22).

5. Derivations. In this section we shall consider the derivations (suitably defined) of the doubly graded algebra of differential forms on a manifold with values in the vector bundles obtained, from a given vector buadle over the manifold, by forming the tensor product of bundles.

The algebraic description is as follows. Let $S=\left\{{ }^{s} S^{p}\right\}, s, p=0,1, \cdots$, be a doubly graded algebra (with unit) over a commutative ring $K$ with unit, that is, $\Phi \Psi \in{ }^{s+t} S^{p+r}$ for $\Phi \in{ }^{s} S^{p}$ and $\Psi \in{ }^{t} S^{r}$. A map $D: \mathcal{S} \rightarrow \mathcal{S}$ will be called a derivation of $S$ of degree $q$ if

(a) $D\left({ }^{8} S^{p}\right) \subset{ }^{8} S^{q+p}$,

(b) $D$ is $K$-linear,

(c) $D(\Phi \Psi)=(D \Phi) \Psi+(-1)^{q p} \Phi(D \Psi)$,

$\Phi \in{ }^{*} S^{p}, \Psi \in \mathcal{S}$.

From these axioms it follows easily that (i) the set of all derivations of $\delta$ of degree $q$ is a $K$-module, (ii) the commutator of two derivations (with signs, according to degrees, as in (39)) is again a derivation whose degree is the sum of the degrees of the two given derivations. Thus the set of all derivations of $\mathcal{S}$ has a structure of graded Lie algebra over $K$. Further, (iii) if $\mathcal{S}$ may also be considered as an algebra over $\widehat{K}$, where $K \subset \widehat{K}$, the subset of derivations of $\mathcal{S}$ which are $\widehat{K}$-linear forms a graded subalgebra of the graded Lie algebra of derivations over $K$, and has a structure of graded Lie algebra over $\widehat{K}$. Finally, (iv) for any derivation $D$ of $\mathcal{S}$ we have $D \Phi=0$ for $\Phi \in K \subset{ }^{0} S^{0}$.

We remark that the restriction of the derivations $D$ and the axioms (a), (b), and (c) to the graded subalgebra ${ }^{0} S^{*} \subset S$ gives the usual derivations in ${ }^{0} S^{*}$. Also, the same axioms define the derivations of degree $q$ of a graded Lie algebra $L^{*}$ over $K$ if (c) is interpreted as

(c') $D[\Phi, \Psi]=[D \Phi, \Psi]+(-1)^{q p}[\Phi, D \Psi]$,

$\Phi \in L^{p}, \Psi \in L^{*}$

and statements (i)-(iii), but not (iv), remain valid in this case.

Given a differentiable vector bundle $B \rightarrow M$, we shall construct sheaves over $M$ for which the algebraic description above applies in the stalks over $x$, for each $x \in M$, with $K=R$ and $\widehat{K}=A_{x}^{0}$. Let ${ }^{s} B \rightarrow M$ denote the vector bundle 
over $M$ which has fibre $\left({ }^{8} B\right)_{x}=B_{x} \otimes \cdots \otimes B_{x}$ (s times), $x \in M$; then ${ }^{1} B \rightarrow M$ is the given bundle and ${ }^{0} B \rightarrow M$ is the product bundle with fibre $R$. Let ${ }^{\circ} S^{p}$ denote the sheaf of germs of differential forms of degree $p$ on $M$ with values in ${ }^{s} B \rightarrow M, s, p=0,1, \cdots$. For $\Phi \in \in^{s} S^{p}$ and $\Psi \in{ }^{t} S^{r}$, the product $\Phi \Psi \in^{s+t} S^{p+r}$ is defined by

$$
\left\langle U_{p+r}, \Phi \Psi\right\rangle=\sum_{I_{p}, J_{r}} \epsilon_{I J}\left\langle U_{I}, \Phi\right\rangle \otimes\left\langle U_{J}, \Psi\right\rangle
$$

Note that this product coincides with the tensor product (of germs of local sections) if $p=r=0$, and with the exterior product if $s=t=0$, by (37). Then $\mathcal{S}=\left\{S^{p}\right\}$ is a doubly graded sheaf of algebras (over $R$ or over $A^{0}$ ), with the graded subalgebra ${ }^{0} S^{*}$ corresponding to the exterior algebra $A^{*}$ of differential forms on $M$.

To define the sheaf of germs of derivations of $\delta$, we first consider, for each open $U \subset M$, the derivations (over $R$ ) of the algebra $\delta_{U}$ of forms defined on $U$. A standard lemma $[2 ; 4]$, which depends on the facts that any such derivation vanishes on $R \subset S_{U}$ and that $A_{U}^{0} \subset S_{U}$, then shows that a derivation in $U$ is a local operator, in the sense that $\Phi=\Psi$ in $V \subset U$, where $V$ is open, implies $D \Phi=D \Psi$ in $V$. Using the obvious restrictions of forms and of derivations to smaller open sets, we then obtain the sheaf $D^{*}$ of germs of derivations of $\mathcal{S}$. The sheaf $D^{*}$ has a structure of graded Lie algebra (over $R$ ), the bracket product being defined by commutators; the subset consisting of derivations which are $A^{0}$-linear is a graded subalgebra of $D^{*}$ and has a structure of graded Lie algebra over $A^{0}$.

The results of Frölicher and Nijenhuis [2] show that the sheaf of germs of derivations of degree $q$ of the exterior algebra $A^{*}$ on $M$ is isomorphic to $B^{q} \oplus_{R} B^{q+1},-1 \leqq q \leqq n=\operatorname{dim} M$ (with $B^{-1}$ defined to be zero), and is zero for other choices of $q$. Here $B^{q}$ is the sheaf of germs of vector-valued $q$-forms on $M$. The derivation of degree $q$ corresponding to $V+W$, with $V \in B^{q}$, $W \in B^{q+1}$, is

$$
D \phi=d_{V} \phi+\phi \pi W, \quad \phi \in A^{*},
$$

where the right-hand member is defined by (29) and (25).

Let $P \rightarrow M$ be the principal bundle with group $G L(m, R)$ of the given vector bundle $B \rightarrow M$, where $m$ is the dimension of the fibre $B_{x}$. Then all the vector bundles ${ }^{\circ} B \rightarrow M$ are associated with $P \rightarrow M$. We shall show that the sheaf $D^{q}$ of germs of derivations (over $R$ ) of the doubly graded sheaf $S=\left\{{ }^{*} S^{p}\right\}$ is isomorphic to $\Sigma^{q} \oplus_{R} B^{q+1},-1 \leqq q \leqq n=\operatorname{dim} M$ (with $\Sigma^{-1}$ defined to be zero), and is zero for other choices of $q$. The sheaf $\Sigma^{q}, 0 \leqq q \leqq n$, is determined by the principal bundle $P \rightarrow M$, as in $\S 3$.

We begin by showing that

$$
\Sigma^{q} \oplus R B^{q+1} \rightarrow D^{q}, \quad-1 \leqq q \leqq n ;
$$


the derivation corresponding to $\sigma+W$, with $\sigma \in \Sigma^{q}, W \in B^{q+1}$, is

$$
D \Phi=\sigma \cdot \Phi+\Phi \pi W,
$$

$\Phi \in \mathcal{S}$,

the right-hand member being defined by (20) and (25). Axioms (a) and (b) are obviously satisfied for the map $D: \varsigma \rightarrow \varsigma$ defined by (51), and it remains to verify (c).

To show that

$$
(\Phi \Psi) \pi W=(\Phi \pi W) \Psi+(-1)^{q p} \Phi(\Psi \pi W)
$$

for $\Phi \in{ }^{s} S^{p}, \Psi \in{ }^{t} S^{r}, W \in B^{q+1}$, we combine (25) and (48) to give

$$
\begin{aligned}
\left\langle U_{q+p+r},(\Phi \Psi) \pi W\right\rangle= & \sum_{H_{q+1}, S_{p+r-1}} \epsilon_{H S}\left\langle\left\langle U_{H}, W\right\rangle \wedge U_{S}, \Phi \Psi\right\rangle \\
= & \sum_{H_{q+1}, M_{p-1}, J_{r}} \epsilon_{H M J}\left\langle\left\langle U_{H}, W\right\rangle \wedge U_{M}, \Phi\right\rangle \otimes\left\langle U_{J}, \Psi\right\rangle \\
& +(-1)^{p} \sum_{H_{q+1}, I_{p}, L_{r-1}} \epsilon_{H I L}\left\langle U_{I}, \Phi\right\rangle \otimes\left\langle\left\langle U_{H}, W\right\rangle \wedge U_{L}, \Psi\right\rangle \\
= & \left\langle U_{q+p+r},(\Phi \pi W) \Psi+(-1)^{q p} \Phi(\Psi \pi W)\right\rangle
\end{aligned}
$$

since $\epsilon_{H I}=(-1)^{q p+p} \epsilon_{I H}$. Next we note that

$$
\sigma \cdot(\Phi \Psi)=(\sigma \cdot \Phi) \Psi+(-1)^{q p} \Phi(\sigma \cdot \Psi), \quad \sigma \in \Sigma^{q}, \Phi \in{ }^{s} S^{p}, \Psi \in{ }^{t} S^{r},
$$

holds for $q=p=r=0$ by the basic definition (12) since $\Phi \Psi=\Phi \otimes \Psi$ in this case. For the general case, we use the property

$$
v \square\left(U_{p} \wedge U_{r}\right)=\left(v \square U_{p}\right) \wedge U_{r}+U_{p} \wedge\left(v \square U_{r}\right), \quad v \in B^{0},
$$

of classical Lie derivatives to compute, using (20) and (48),

$$
\begin{aligned}
\left\langle U_{q+p+r},\right. & \sigma \cdot(\Phi \Psi)\rangle \\
= & \sum_{I_{q}, S_{p+r}} \epsilon_{I S}\left\{\left\langle U_{I}, \sigma\right\rangle \cdot\left\langle U_{S}, \Phi \Psi\right\rangle-\left\langle\left\langle U_{I}, \pi \sigma\right\rangle \square U_{S}, \Phi \Psi\right\rangle\right\} \\
& +(-1)^{q-1} \sum_{K_{2}, L_{q-1}, R_{p+r-1}} \epsilon_{K L R}\left\langle\left\langle\left[U_{K}\right] \wedge U_{L}, \pi \sigma\right\rangle \wedge U_{R}, \Phi \Psi\right\rangle \\
= & \sum_{I_{q}, J_{p}, P_{r}} \epsilon_{I J P}\left\{\left\langle U_{I}, \sigma\right\rangle \cdot\left(\left\langle U_{J}, \Phi\right\rangle \otimes\left\langle U_{P}, \Psi\right\rangle\right)\right. \\
& \left.-\left\langle\left\langle U_{I}, \pi \sigma\right\rangle \square U_{J}, \Phi\right\rangle \otimes\left\langle U_{P}, \Psi\right\rangle-\left\langle U_{J}, \Phi\right\rangle \otimes\left\langle\left\langle U_{I}, \pi \sigma\right\rangle \square U_{P}, \Psi\right\rangle\right\} \\
& +(-1)^{q-1} \sum_{K_{2}, L_{q-1}, M_{p-1}, P_{r}} \epsilon_{K L M P}\left\langle\left\langle\left[U_{K}\right] \wedge U_{L}, \pi \sigma\right\rangle \wedge U_{M}, \Phi\right\rangle \otimes\left\langle U_{P}, \Psi\right\rangle \\
& +(-1)^{q-1+p} \sum_{K_{2}, L_{q-1}, J_{p}, T_{r-1}} \epsilon_{K L J T}\left\langle U_{J}, \Phi\right\rangle \otimes\left\langle\left\langle\left[U_{K}\right] \wedge U_{L}, \pi \sigma\right\rangle \wedge U_{T}, \Psi\right\rangle .
\end{aligned}
$$

We then expand the terms $\left\langle U_{I}, \sigma\right\rangle \cdot\left(\left\langle U_{J}, \Phi\right\rangle \otimes\left\langle U_{P}, \Psi\right\rangle\right)$ by the special case of (52) already checked, and use $\epsilon_{I J}=(-1)^{q p} \epsilon_{J I}$, etc., to verify that the above terms give $\left\langle U_{q+p+r},(\sigma \cdot \Phi) \Psi+(-1)^{q p} \Phi(\sigma \cdot \Psi)\right\rangle$. 
To complete the proof we must show that the correspondence (50) which is obviously injective and $R$-linear, is also surjective, $-1 \leqq q \leqq n$, and that $D^{q}=0$ for other choices of $q$. We use the fact that any derivation $D \in D^{*}$ is determined uniquely by its action on a set of generators (over $R$ ) of $\mathcal{S}$, because of properties (b) and (c). Clearly $S$ is generated over $R$ by the elements of $A^{*}={ }^{0} S^{*}$ and of ${ }^{1} S^{0}$. A derivation of degree $q$ vanishes on $A^{*}$ unless $-1 \leqq q$ $\leqq n$, by [2] , and on ${ }^{1} S^{0}$ unless $0 \leqq q \leqq n$, by (a) and the fact that ${ }^{1} S^{p}=0$ unless $0 \leqq p \leqq n$. Thus we conclude that $D^{q}=0$ unless $-1 \leqq q \leqq n$. For given $D \in D^{q}$ with $-1 \leqq q \leqq n$, we have

$$
D \phi=d_{V} \phi+\phi \pi W, \quad \phi \in A^{*},
$$

with $V \in B^{q}, W \in B^{q+1}$, by [2]. Since locally we may always choose as generators the elements of $A^{*}$ together with a basis (over $A^{0}$ ) for ${ }^{1} S^{0}$, say $\Phi_{(\rho)}$, $\rho=1, \cdots, m$, it is sufficient to show that there exists a (unique) $\sigma \in \Sigma^{a}$ satisfying

$$
D \Phi_{(\rho)}=\sigma \cdot \Phi_{(\rho)}, \quad \pi \sigma=V, \quad \rho=1, \cdots, m .
$$

(Note that $\Phi_{(\rho)} \pi W=0$.) If $\sigma$ is represented locally by $V=\pi \sigma \in B^{q}$ and an $(m \times m)$-matrix $\left(\sigma_{\nu}^{\mu}\right)$ of $q$-forms, and $\Phi_{(\rho)}$ by functions $\Phi_{(\rho)}^{\mu}$, then by (23) we must solve the system

$$
\left(D \Phi_{(\rho)}\right)^{\mu}=d_{V} \Phi_{(\rho)}^{\mu}-\sigma_{\nu}^{\mu} \Phi_{(\rho)}^{\nu}, \quad \quad \mu, \rho=1, \cdots, m,
$$

for the $q$-forms $\sigma_{\nu}^{\mu}$. The existence and uniqueness of the solution follows immediately from the fact that the matrix $\left(\Phi_{(\rho)}^{\mu}\right)$ is nonsingular if $\left\{\Phi_{(\rho)}\right\}$ is a (local) basis for ${ }^{1} S^{0}$. It is also clear that we obtain $\sigma=0$ if $q=-1$. From (53) and (54) it follows that $\sigma+W$ corresponds to the given $D$ in (50) and that

$$
\Sigma^{q} \oplus R B^{q+1} \approx D^{q}, \quad-1 \leqq q \leqq n,
$$

as was to be shown.

In using (55) to express (54) we have assumed that the action of the group $G L(m, R)$ on the fibre $F$ of $B \rightarrow M$ is the obvious one. The same conclusion holds if we consider the dual action of $G L(m, R)$. Equivalently, the same sheaf $D^{*}$ of germs of derivations is obtained if we start from the dual bundle $B^{*} \rightarrow M$, associated with the same principal bundle, rather than from $B \rightarrow M$.

More generally we may consider derivations of the triply graded sheaf $S=\left\{{ }_{i}^{s} S^{p}\right\}$, where ${ }_{0}^{1} S^{0}$ is the sheaf of germs of sections of $B \rightarrow M$ and ${ }_{1}^{0} S^{0}$ is the sheaf of germs of sections of $B^{*} \rightarrow M$, with multiplication defined as in (48). If we add the condition that $D \in D^{*}$ is determined, by duality, on ${ }_{1}^{0} S^{0}$ by its action on $A^{*}$ and ${ }_{0}^{1} S^{0}$, the same sheaf $D^{*}$ of germs of derivations is obtained. For $D$ of degree 0 , this condition should reduce to the usual condition that derivations in the mixed tensor algebra shall commute with tensorial contraction. Let $\Phi \bigvee \Psi \in \in_{0}^{0} S^{0}=A^{0}$ denote the tensorial contraction, induced 
by duality, of $\Phi \in_{1}^{0} S^{0}$ and $\Psi \in_{0}^{1} S^{0}$. For $\Phi \in_{1}^{0} S^{p}$ and $\Psi \in_{0}^{1} S^{r}$, we define $\Phi \bigvee \Psi$ $\in_{0}^{0} S^{p+r}=A^{p+r}$ by

$$
\left\langle U_{p+r}, \Phi \bigvee \Psi\right\rangle=\sum_{I_{p}, J_{r}} \epsilon_{I J}\left\langle U_{I}, \Phi\right\rangle \bigvee\left\langle U_{J}, \Psi\right\rangle .
$$

The additional axiom for $D \in D^{q}$ is then

$$
D(\Phi \bigvee \Psi)=D \Phi \bigvee \Psi+(-1)^{q p} \Phi \bigvee D \Psi,
$$$$
\Phi \in{ }_{1}^{0} S^{p}, \Psi \in{ }_{0}^{1} S^{*} .
$$

Actually, it is sufficient to require only that (d) hold for $\Phi \in{ }_{1}^{0} S^{0}$ and $\Psi \in_{0}^{1} S^{0}$; the extended definition (56) is still needed in order that the right-hand side in (d) be defined if $q>0$.

We have noted that the graded sheaf

$$
D^{*} \approx \Sigma^{*} \oplus R B^{*}
$$

has a structure of graded Lie algebra (over $R$ ) induced by forming the commutators of derivations. The graded sheaf $B^{*}$ corresponds to a graded subalgebra (over $A^{0}$ ) of $D^{*}$, as is seen by writing (26) in the form

$$
(\Phi \pi W) \pi V-(-1)^{a r}(\Phi \pi V) \pi W=\Phi \pi\left\{(W \pi V)-(-1)^{a r}(V \pi W)\right\},
$$

for $\Phi \in \mathcal{S}, V \in B^{q+1}, W \in B^{r+1}$; this shows that the commutator of two contractions, derivations of degrees $q$ and $r$ respectively, is again a contraction. The graded sheaf $\Sigma^{*}$ corresponds to a graded subalgebra (over $R$ ) of $D^{*}$ in which the commutator coincides with the bracket product defined by $(45)$, because of (39); in particular, we see that $\Sigma^{0}$ determines a subalgebra (over $R$ ) of $D^{*}$, that $\Xi^{*} C \Sigma^{*}$ determines a graded subalgebra (over $A^{0}$ ) of $D^{*}$, and that $\Xi^{0}$ determines a subalgebra (over $A^{0}$ ) of $D^{*}$.

By considering the commutators of derivations of the two types, we find the identities

$$
(\sigma \cdot \Phi) \pi V-(-1)^{q s} \sigma \cdot(\Phi \pi V)=(\sigma \pi V) \cdot \Phi+(-1)^{q} \Phi \pi[V, \pi \sigma],
$$

valid for $\sigma \in \Sigma^{q}, V \in B^{s+1}, \Phi \in \mathcal{S}$, and

$$
\begin{aligned}
{[\sigma, \tau] \pi V=} & {[\sigma \pi V, \tau]+(-1)^{q s}[\sigma, \tau \pi V] } \\
& -(-1)^{q s} \tau \pi[\pi \sigma, V]+(-1)^{r(q+s)} \sigma \pi[\pi \tau, V],
\end{aligned}
$$

valid for $\sigma \in \Sigma^{q}, \tau \in \Sigma^{r}, V \in B^{s+1}$; all terms in (58) are elements of $\Sigma^{q+r+s}$.

In fact, since both sides of (57) represent derivations of $S$ applied to an arbitrary $\Phi \in \delta$, it is sufficient to verify that these derivations coincide on the generators $A^{*}$ and ${ }_{0}^{1} S^{0}$ of $\mathrm{S}$. For $\Phi \in A^{*}$, this identity has been proved by Frölicher and Nijenhuis [2]. For $\Phi \in_{0}^{1} S^{0}$, the identity reduces to

$$
(\sigma \cdot \Phi) \pi V=(\sigma \pi V) \cdot \Phi
$$

where $\sigma \in \Sigma^{q}$ and $V \in B^{s+1}$ are arbitrary. Combining (20) and (25), we find trivially 


$$
\begin{aligned}
\left\langle U_{q+s},(\sigma \cdot \Phi) \pi V\right\rangle & =\sum_{T_{c+1}, L_{q-1}} \epsilon_{T L}\left\langle\left\langle U_{T}, V\right\rangle \wedge U_{L}, \sigma \cdot \Phi\right\rangle \\
& =\sum_{T_{q+1}, L_{q-1}} \epsilon_{T L}\left\langle\left\langle U_{T}, V\right\rangle \wedge U_{L}, \sigma\right\rangle \cdot \Phi \\
& =\left\langle U_{q+s},(\sigma \pi V) \cdot \Phi\right\rangle,
\end{aligned}
$$

which proves (59) and therefore (57). The identity (58) has also been given by Frölicher and Nijenhuis in the case that the derivations act on $\Phi \in A^{*}$. For $\Phi \in_{0}^{1} S^{0}$ we have, by (39) and (59),

$$
\begin{aligned}
([\sigma, \tau] \pi V) \cdot \Phi & =([\sigma, \tau] \cdot \Phi) \pi V \\
& =(\sigma \cdot(\tau \cdot \Phi)) \pi V-(-1)^{q r}(\tau \cdot(\sigma \cdot \Phi)) \pi V .
\end{aligned}
$$

Also, by using (57) twice, we find

$$
\begin{aligned}
(\sigma \cdot(\tau \cdot \Phi)) \pi V= & (\sigma \pi V) \cdot(\tau \cdot \Phi)+(-1)^{g s} \sigma \cdot((\tau \pi V) \cdot \Phi) \\
& -(-1)^{q s}(\tau \pi[\pi \sigma, V]),
\end{aligned}
$$

and a similar formula for $(\tau \cdot(\sigma \cdot \Phi)) \pi V$. These formulas combine easily to give (58) for $\Phi \in_{0}^{1} S^{0}$ and therefore for all $\Phi \in \mathcal{S}$. We remark that (58) shows that any contraction by an element of $B^{*}$ defines a derivation (over $A^{0}$ ) of the graded Lie algebra $\Xi^{*} \subset \Sigma^{*}$ (but not of $\Sigma^{*}$ ).

Whenever the group of the principal bundle of the given vector bundle $B \rightarrow M$ can be reduced from $G L(m, R)$ to a closed subgroup $G \subset G L(m, R)$, another graded subalgebra of $D^{*}$ is defined. In fact, the argument which proves (50) does not use the fact that the principal bundle has group $G L(m, R)$, and we have

$$
0 \rightarrow \Sigma^{*} \oplus R B^{*} \rightarrow D^{*}
$$

for any choice of the principal bundle $P \rightarrow M$ of the vector bundle $B \rightarrow M$. However, any derivation $D$ for which the uniquely determined local $\mathfrak{g l}(m, R)$ valued form $\left(\sigma_{\nu}^{\mu}\right)$ in (55) is not $\mathrm{g}$-valued cannot be obtained from an element in $\Sigma^{*} \oplus_{R} B^{*}$. To verify that $\Sigma^{*} \oplus_{R} B^{*}$ determines a graded subalgebra of $D^{*}$, we need only check that the commutator of the derivations corresponding to $\sigma \in \Sigma^{*}$ and $V \in B^{*}$ lies in the image of $\Sigma^{*} \oplus_{R} B^{*}$. This follows from the fact that the right-hand member of (57) clearly represents a derivation which lies in this image.

The proofs of (57) and (58) given here cover only the case that $P \rightarrow M$ has group $G \subset G L(m, R)$ and the operators act on $\Phi \in \mathcal{S}$. These identities are valid in general and may be proved by direct computation.

6. Connections. A connection in the principal bundle $P \rightarrow M$ is a splitting of the corresponding exact sequence (1) of vector bundles [1]. Such a splitting can be expressed in two ways: by an injective (differentiable) mapping

$$
\chi: \quad T(M) \rightarrow T(P) / G
$$


such that $\pi \circ \chi$ is the identity mapping on $T(M)$ and such that

$$
\chi_{x}: \quad(T(M))_{x} \rightarrow(T(P) / G)_{x}
$$

is linear for each $x \in M$; or by a surjective (differentiable) mapping

$$
\Pi: \quad T(P) / G \rightarrow K
$$

such that $\iota \circ \Pi$ is the identity mapping on $K$ and such that

$$
\Pi_{x}: \quad(T(P) / G)_{x} \rightarrow K_{x}
$$

is linear for each $x \in M$. If $\chi$ and $\Pi$ correspond to the same splitting, then the sequence

$$
0 \rightarrow T(M) \stackrel{\chi}{\rightarrow} T(P) / G \stackrel{\Pi}{\rightarrow} K \rightarrow 0
$$

is exact.

Because of (60), a mapping $\chi$ may be described as a globally defined 1form on $M$ with values in the vector bundle $T(P) / G$, satisfying $\pi \chi=\delta$. The image of $u \in T(M)$ under $\chi$ may then be denoted by $\chi \pi u$. The usual local descriptions follow from the isomorphism of the bundle $T(P) / G \rightarrow T(M)$ with the bundle $T(P) \times_{T(G)} \mathfrak{g} \rightarrow T(M)$. If $\left\{U_{i}\right\}$ is an open covering of $M$ such that $T(P) / G \mid T\left(U_{i}\right)$ is isomorphic to $T\left(U_{i}\right) \times g$, then $\chi$ may be expressed by $\left(\pi \chi=\delta\right.$ and) a collection $\left\{\chi_{i}\right\}$ of $g$-valued 1 -forms $\chi_{i}$ defined on $U_{i}$, the values in $U_{i} \cap U_{k}$ being related by the transition laws (3). Still more explicitly, if $G \subset G L(m, R)$, then $\chi$ is represented locally by (a pair consisting of $\pi \chi=\delta$ and) an $(m \times m)$-matrix of 1 -forms, denoted classically by $\left(\omega_{\nu}^{\mu}\right)$ or by $\left(-\Gamma_{\nu \alpha}^{\mu}(x) d x^{\alpha}\right)$, where $\left(x^{1}, \cdots, x^{n}\right)$ are local coordinates on $M$. The transition law for the Christoffel symbols $\Gamma_{\nu \alpha}^{\mu}$ is obtained from (3) if we take coordinates $\left(x^{1}, \cdots, x^{n}\right)$ in $U_{i}$ and $\left(y^{1}, \cdots, y^{n}\right)$ in $U_{k}$, with

$$
u=\frac{\partial}{\partial y^{\beta}}=\frac{\partial x^{\alpha}}{\partial y^{\beta}} \frac{\partial}{\partial x^{\alpha}}
$$

and

$$
\lambda_{i}=\chi_{i} \pi u=\left(-\Gamma_{\nu \alpha}^{\mu} \frac{\partial x^{\alpha}}{\partial y^{\beta}}\right), \quad \lambda_{k}=\chi_{k} \pi u=\left(-\tilde{\Gamma}_{\nu \beta}^{\mu}\right) ;
$$

the usual case corresponds to $G=G L(n, R), n=\operatorname{dim} M$, with

$$
g_{i k}=\left(\frac{\partial x^{\mu}}{\partial y^{v}}\right) \text {. }
$$

A splitting of the sequence (1) defined by a mapping $\Pi: T(P) / G \rightarrow K$ may be expressed in terms of a globally defined $g$-valued 1 -form $\Omega$ on $P$, that is, a 1 -form on $P$ with values in the product bundle $P \times g$, such that $\Omega$ induces 
a mapping $\Pi$ from the quotient $T(P) / G$ of $T(P)$ into $K=P \times_{G} \mathfrak{g}$, a quotient

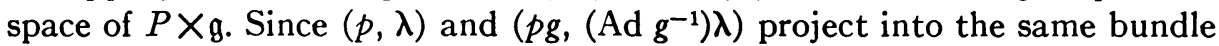
point of $K$, for $p \in P, g \in G, \lambda \in \mathfrak{g}$, this means that $\Omega$ must satisfy

$$
\Omega(t g)=\left(\operatorname{Ad} g^{-1}\right) \Omega(t), \quad t \in T(P), g \in G .
$$

The form $\Omega$ must also satisfy a further condition to ensure that $\Pi \circ \iota$ is the identity mapping on $K$.

The classical notion of covariant differentiation, corresponding to a connection $\chi$ in the principal bundle $P \rightarrow M$, is expressed in terms of the basic operation of $\S 2$ as follows. Let $u$ be a tangent vector to $M$ at $x$, and let $s$ be a section of a vector bundle $B \rightarrow M$, associated with $P \rightarrow M$, defined at least on a curve through $x$ having tangent $u$ at $x$. Then the covariant derivative $D_{u} s$ of $s$ in the direction $u$ is given by (12) with $\sigma=\chi \pi u$. For this particular choice of $\sigma$ (depending on $\chi$ and on $u$ ), the vector $j_{*}\left(\sigma_{p}, 0\right)$, tangent to $B$ at $s(x)$ and lying over $u$, is called the "parallel" to $u$ at $s(x)$; the geometric interpretation of the basic operation shows that $D_{u} s$ is obtained from the difference between the tangent to $s$ at $s(x)$, lying over $u$, and the parallel to $u$ at $s(x)$. The classical coordinate representation is obtained by setting $\sigma=\chi \pi u$ in (15) (or (16) or (17), etc.) to give

$$
\left(D_{u} s\right)^{\mu}=((\chi \pi u) \cdot s)^{\mu}=u^{\alpha}\left(\frac{\partial f^{\mu}}{\partial x^{\alpha}}+\Gamma_{\nu \alpha}^{\mu} f^{\nu}\right),
$$

where $s$ has fibre coordinates $f^{\mu}$ and $u=u^{\alpha} \partial / \partial x^{\alpha}$.

It is easily verified that a splitting of the exact sequence (1) of vector bundles is equivalent to a splitting (over $A^{0}$ ) of the exact sequence (18), for $q=0$. We then consider the connection form $\chi$ as an element of $H I^{0}\left(M, \Sigma^{1}\right)$ with $\pi \chi=\delta \in H^{0}\left(M, B^{1}\right)$. It is evident that the connections in a given principal bundle are in one-one correspondence with the subset of $H^{0}\left(M, \Sigma^{1}\right)$ consisting of forms which project into $\delta$ under $\pi$. Since a differentiable manifold is paracompact (by definition), a connection always exists, for any choice of the principal bundle $P \rightarrow M$. If $\chi$ is a connection in the given principal bundle, then all other connections are of the form $\chi+\xi$ where $\xi \in H^{0}\left(M, \Xi^{1}\right)$. In particular, if $P \rightarrow M$ is the trivial principal bundle, there is one and only one connection, viz. $\chi=\delta$.

The curvature form $R$ corresponding to a given connection $\chi$ is defined by

$$
R=\frac{1}{2}[\chi, \chi]
$$

then $R \in H^{0}\left(M, \Xi^{2}\right)$ by (42) and the fact [2] that

$$
[\delta, V]=0, \quad V \in B^{*} .
$$

The covariant derivative is defined by 


$$
D \Phi=\chi \cdot \Phi,
$$

$\Phi \in S^{*}$,

for arbitrary choices of the associated vector bundle $B \rightarrow M$ determining $S^{*}$. Then, by (39) and (40), we have

$$
R \cdot \Phi=\chi \cdot(\chi \cdot \Phi)=D^{2} \Phi .
$$

For $G \subset G L(m, R)$, the curvature form $R$ is uniquely determined by the property (64).

An explicit computation of (61) according to the definition (45) gives, for $u, v \in B^{0}$,

$$
\begin{aligned}
\langle u \wedge v, R\rangle & =\left\langle u \wedge v, \frac{1}{2}[\chi, \chi]\right\rangle \\
& =[\langle u, x\rangle,\langle v, x\rangle]-\langle[u, v], \chi\rangle \\
& =[\chi \pi u, \chi \pi v]-\chi \pi[u, v] .
\end{aligned}
$$

That is, the curvature form $R$ measures the extent to which $\chi: B^{0} \rightarrow \Sigma^{0}$ fails to be a Lie algebra homomorphism. In particular, the curvature of a connection vanishes if and only if the connection, which is a splitting of the exact sequence

$$
0 \rightarrow \Xi^{0} \stackrel{\iota}{\rightarrow} \Sigma^{0} \stackrel{\pi}{\rightarrow} B^{0} \rightarrow 0
$$

of sheaves of $A^{0}$-modules, also induces a splitting of (66) considered as an exact sequence of sheaves of Lie algebras over $\boldsymbol{R}$.

A connection $\chi$ induces a splitting of the sequences (18), $q=0,1, \cdots, n$ $=\operatorname{dim} M$, by defining

$$
\chi: \quad B^{q} \rightarrow \Sigma^{q}
$$

to be the map which sends $V \in B^{q}$ into $\chi \pi V \in \Sigma^{q}$, since

$$
\pi(\chi \pi V)=\pi \chi \pi V=\delta \pi V=V .
$$

Next we note that

$$
R \pi V=[\chi \pi V, \chi]
$$

as is seen by taking $\sigma=\tau=\chi$ in (58) and using (61), (62), and (40). Then we compute, for $V \in B^{q}, W \in B^{r}$, again using (58),

$$
\begin{aligned}
(R \pi V) \pi W-R \pi(V \pi W)= & {[\chi \pi V, \chi] \pi W-[\chi \pi(V \pi W), \chi] } \\
= & {[(\chi \pi V) \pi W, \chi]+(-1)^{q(r-1)}[\chi \pi V, \chi \pi W] } \\
& -(-1)^{q(r-1)} \chi \pi[V, W]-[\chi \pi(V \pi W), \chi]
\end{aligned}
$$

or, since $\chi$ is of degree 1 , 


$$
\begin{aligned}
{[\chi \pi V, \chi \pi W]-\chi \pi[V, W] } & =(-1)^{q(r-1)}\{(R \pi V) \pi W-R \pi(V \pi W)\} \\
& =(-1)^{r-1}\{(R \pi W) \pi V-R \pi(W \pi V)\}
\end{aligned}
$$

using (26). This formula reduces to (65) if $q=r=0$ since then $(R \pi V) \pi W$ $=\langle V \wedge W, R\rangle$ and $V \pi W=0$. From (67) we conclude that no additional curvature forms need be introduced to express the extent to which $\chi: B^{*} \rightarrow \Sigma^{*}$ fails to be a homomorphism of graded Lie algebras and, in particular, that a connection induces a splitting of the exact sequence (46) of sheaves of graded Lie algebras if and only if the corresponding curvature form vanishes.

For $V \in B^{q}, \Phi \in S^{p}$, we define the absolute derivative $D_{V} \Phi$ of $\Phi$ with respect to $V$ by

$$
D_{V} \Phi=(\chi \pi V) \cdot \Phi
$$

then $D_{V} \Phi \in S^{q+p}$. By (28), the covariant derivative coincides with $D_{\delta}$. Choosing $\sigma=\chi$ in (57), we obtain

$$
(\chi \pi V) \cdot \Phi=(\chi \cdot \Phi) \pi V+(-1)^{q} \chi \cdot(\Phi \pi V),
$$

$V \in B^{q}$,

or

$$
D_{V} \Phi=(D \Phi) \pi V+(-1)^{q} D(\Phi \pi V),
$$$$
V \in B^{q} .
$$

From (24) and (29), we see that the absolute derivative $D_{V}$ coincides with the (generalized) Lie derivative $d_{V}$ when the associated vector bundle is the trivial bundle with fibre $R$, and that the covariant derivative induces the exterior derivative in this case. In general, we have

$$
D_{V} D_{W}-(-1)^{a r} D_{W} D_{V} \neq D_{[V, W]}, \quad V \in B^{q}, W \in B^{r},
$$

the difference being an operator in $\Xi^{a+r}$ given by the right-hand member of (67) which vanishes, in general, only if $R=0$, i.e., if $D^{2}=0$.

Finally, we note that a connection $\chi$ induces a derivation $D$ of degree 1 in the graded Lie algebra $\Sigma^{*}$, by setting

$$
D_{\sigma}=[\chi, \sigma], \quad \sigma \in \Sigma^{*} .
$$

In fact, for $\sigma \in \Sigma^{q}, \tau \in \Sigma^{*}$, we have

$$
D[\sigma, \tau]=[D \sigma, \tau]+(-1)^{q}\left[\sigma, D_{\tau}\right]
$$

by taking $\omega=\chi$ in (41). Moreover,

$$
\begin{gathered}
D^{2} \sigma=[R, \sigma], \\
D_{\chi}=2 R,
\end{gathered}
$$

and

$$
D R=0,
$$

(second) Bianchi identity. These also follow from (41) together with (61). 
The operator $D$ satisfies

$$
D \Sigma^{*} \subset \Xi^{*}
$$

and induces the zero derivation on $B^{*}$, by (62) and (42).

The obvious generalization

$$
D_{V} \sigma=[\chi \pi V, \sigma], \quad V \in B^{*}, \sigma \in \Sigma^{*},
$$

satisfies the analogue of (72), again by (41), but does not satisfy the analogue of (70) (except when restricted to $\Xi^{*}$ ). In fact, it follows from (58) that

$$
D_{V} \sigma=\left(D_{\sigma}\right) \pi V+(-1)^{q} \boldsymbol{D}(\sigma \pi V) \pm \chi \pi[\pi \sigma, V], \quad V \in B^{q}, \sigma \in \Sigma^{*} .
$$

For the restriction to $\Xi^{*}$, we have

$$
D_{V} \xi=D_{V} \xi
$$$$
V \in B^{*}, \xi \in \Xi^{*} \text {. }
$$

In particular, the (second) Bianchi identity (75) may equally well be stated as

$$
D R=0,
$$

since $R \in H^{0}\left(M, \Xi^{2}\right)$. The fact that $D_{V} \xi$ is defined follows from the fact that the bundle $K \rightarrow M$ is an associated vector bundle of the given principal bundle (with fibre $\mathfrak{g}$ where $G$ acts on $\mathfrak{g}$ by the adjoint action). The identity (76) is easily verified by representing both members of (76) locally, using (47) for the left-hand side and, for the right-hand side, the analogue of (23) which corresponds to the adjoint action of $G$.

Again,

$$
D_{V} D_{W}-(-1)^{q r} D_{W} D_{V} \neq D_{[V, W]}, \quad V \in B^{q}, W \in B^{r},
$$

in general, the difference depending on the curvature form $R$ of the connection $\chi$. An equivalent statement is the following. For the direct sum decomposition

$$
\Sigma^{*} \approx B^{*} \oplus_{A^{*}} \Xi^{*}
$$

determined by the connection $\chi$, where

with

$$
\sigma \rightarrow V+\xi
$$

$$
V=\pi \sigma, \quad \xi=\sigma-\chi \pi \sigma,
$$

the subspace $B^{*}$ does not form a subalgebra, with respect to the graded Lie algebra structure (over $R$ ) induced from $\Sigma^{*}$, unless the curvature vanishes. Rather, the induced bracket product of $V$ and $W \in B^{*}$ has component $[V, W] \in B^{*}$ and a component in $\Xi^{*}$ given by the right side of (67).

7. Tangential structure. In this section we shall consider the case that the given principal bundle $P \rightarrow M$ is the principal bundle of the tangent bundle of 
$M$; the associated vector bundles are then the tensor bundles on $M$. In the notation of $\$ 5$, with $B \rightarrow M$ denoting the tangent bundle of $M$, the sheaf ${ }_{i}^{s} S^{p}$ denotes the sheaf of germs of $p$-forms on $M$ with values in the bundle of tensors which are contravariant of order $s$ and covariant of order $t$.

In general, the group of $P \rightarrow M$ is $G L(n, R)$, where $n=\operatorname{dim} M$. If the group of the principal bundle of the tangent bundle of $M$ has been reduced to a closed subgroup $G \subset G L(n, R)$, then $M$ is said to carry a $G$-structure subordinate to its differentiable structure. In this case, $P \rightarrow M$ will denote the principal bundle with group $G$ of the tangent bundle of $M$. We shall assume that

$$
\Sigma^{q} \oplus R B^{q+1} \approx D^{q}
$$

in all cases; this means, in the case of a $G$-structure on $M$ (with $G \neq G L(n, R)$ ), that $D^{*}$ has been redefined to denote a graded subsheaf of germs of derivations (determined by the $G$-structure on $M$ ) rather than the graded sheaf of germs of all derivations of $\mathcal{S}$ (which is determined by the differentiable structure on $M$ ).

In the present case, as compared with the general case considered in $\$ 5$, we have certain canonical identifications among the sheaves involved. The typical isomorphisms are

$$
\mu: \quad B^{q} \rightarrow{ }_{a}^{1} S^{q}, \quad q=0,1, \cdots, n=\operatorname{dim} M,
$$

and

$$
\rho:{ }_{1}^{0} S^{0} \rightarrow{ }_{0}^{0} S^{1}=A^{1} .
$$

The two types of contraction, by elements of $B^{*}$, as in (25), or of ${ }_{0}^{1} S^{*}$, as in (56), are related by

$$
(\rho \Phi) \pi V=\Phi \bigvee(\mu V), \quad \Phi \in{ }_{1}^{0} s^{0}, V \in B^{*} .
$$

The natural extension

$$
\rho: \quad{ }_{1}^{0} S^{p} \rightarrow{ }_{0}^{0} S^{p+1}=A^{p+1}
$$

of $(80)$ is defined by

$$
\begin{array}{rlrl}
\left\langle U_{p+1}, \rho \Phi\right\rangle & =\sum_{J_{p}, s} \epsilon_{J_{s}}\left\langle u_{s}, \rho\left\langle U_{J}, \Phi\right\rangle\right\rangle \\
& =\sum_{J_{p}, \varepsilon} \epsilon_{J_{s}}\left\langle U_{J}, \Phi\right\rangle \bigvee\left(\mu u_{s}\right), &
\end{array}
$$

but is not an isomorphism if $p>0$. It is easily verified that the identity (81) is replaced by

$$
(\rho \Phi) \pi V-\rho(\Phi \pi V)=(-1)^{q p} \Phi \bigvee(\mu V), \quad \Phi \in{ }_{1}^{0} S^{p}, V \in B^{q+1},
$$


which reduces to (81) for $p=0$. It is evident from (84) that a derivation of $S$ corresponding to contraction by a vector-valued form $V$ is not compatible with tangential structure. Analogously, we have

$$
\sigma \cdot(\rho \Phi)-\rho(\sigma \cdot \Phi)=(-1)^{q p} \Phi \bigvee(\sigma \cdot \mu \delta), \quad \Phi \in{ }_{1}^{0} S^{p}, \sigma \in \Sigma^{q},
$$

where $\delta \in H^{0}\left(M, B^{1}\right)$ is defined by (27). In fact, for $V=\delta$, the identity (84) becomes

$$
\rho \Phi=\Phi \bigvee \mu \delta, \quad \Phi \in{ }_{1}^{0} S^{*},
$$

using (28). Then, by property (d) of $\S 5$, we have, for $\sigma \in \Sigma^{q}, \Phi \in{ }_{1}^{0} S^{p}$,

$$
\begin{aligned}
\sigma \cdot(\rho \Phi) & =\sigma \cdot(\Phi \bigvee \mu \delta)=(\sigma \cdot \Phi) \bigvee \mu \delta+(-1)^{q p} \Phi \bigvee(\sigma \cdot \mu \delta) \\
& =\rho(\sigma \cdot \Phi)+(-1)^{q p} \Phi \bigvee(\sigma \cdot \mu \delta) .
\end{aligned}
$$

From (85) and (84) we conclude that a derivation $D \in D^{q}$ of $\mathcal{S}$, corresponding to $\sigma+W \in \Sigma^{q} \oplus_{R} B^{q+1}$, is compatible with tangential structure, that is, satisfies

$$
D \rho=\rho D,
$$

if and only if

$$
\mu W=-\sigma \cdot \mu \delta .
$$

(No further conditions are introduced if we consider the fact that the elements ${ }_{0}^{0} S^{p}=A^{p}$ are to be identified with the elements of ${ }_{p}^{0} E^{0}$, for which all operations are also defined. Here ${ }_{p}^{0} E^{0}$ is defined in the same way as ${ }_{p}^{0} S^{0}$, in $\S 5$, using the exterior product in place of the tensor product to construct the associated vector bundle. This is because all constructions, operations, and identifications are determined canonically from the case ${ }_{1}^{0} E^{0}={ }_{1}^{0} S^{0}$ considered above.)

We define the torsion form $J \sigma$ of $\sigma \in \Sigma^{*}$ by

$$
\Im \sigma=\mu^{-1}(\sigma \cdot \mu \delta) ;
$$

the map

$$
\text { J: } \Sigma^{q} \rightarrow B^{q+1}
$$

defined in this way is clearly $R$-linear. The restriction

$$
\text { J: } \Xi^{q} \rightarrow B^{q+1}
$$

is even $A^{0}$-linear, since $\xi \cdot \delta$ does not involve any actual differentiation, $\xi \in \Xi$. We set

$$
L^{*}=\operatorname{ker} J=\left\{\sigma \mid \sigma \in \Sigma^{*}, \sigma \cdot \mu \delta=0\right\}
$$

and 


$$
\Lambda^{*}=\operatorname{ker} \mathfrak{J} \cap \operatorname{ker} \pi=L^{*} \cap \Xi^{*} .
$$

Then $L^{*}$ is a graded subalgebra of the graded Lie algebra $\Sigma^{*}$, but not a graded ideal in $\Sigma^{*}$, and $\Lambda^{*}$ is a graded subalgebra (over $A^{0}$ ) of $\Xi^{*}$, and of $\Sigma^{*}$, but not a graded ideal. However, $\Lambda^{*}$ is a graded ideal in $L^{*}$.

Let $\sigma \in \Sigma^{q}$ be represented locally by $\pi \sigma \in B^{q}$ and an $(n \times n)$-matrix $\left(\sigma_{\nu}^{\mu}\right)$ of $q$-forms, and let $\mu \delta \in_{0}^{1} S^{1}$ be represented by the 1 -forms $\omega^{\mu}, \mu=1, \cdots, n$; for this local representation we have (using $B^{q} \approx B^{0} \otimes{ }^{0} A^{q}$ )

$$
\delta=u_{\mu} \otimes \omega^{\mu},
$$

where $u_{\mu} \in B^{0}$ and $\left\{u_{\mu}\right\}$ is the dual basis to $\left\{\omega^{\mu}\right\}$. Then $\Xi \sigma$ is expressed by

$$
J \sigma=u_{\mu} \otimes(\Im \sigma)^{\mu}
$$

where

$$
(J \sigma)^{\mu}=d_{\pi \sigma} \omega^{\mu}-\sigma_{\nu}{ }_{\nu} \wedge \omega^{\prime},
$$

by (88) and (23).

Let $D_{0}^{*}$ denote the subsheaf of $D^{*}$ consisting of germs of derivations compatible with tangential structure. It is easily verified from the condition (86), which defines $D_{0}^{*}$, that $\mathcal{D}_{0}^{*}$ is a graded subalgebra of the graded Lie algebra $D^{*}$. Combining (87) and (88), we see that under the isomorphism (78) the elements of $D_{0}^{q}$ correspond to elements of the form $\sigma-J \sigma$, with $\sigma \in \Sigma^{q}$, $\exists \sigma \in B^{q+1}$. Thus we have an $R$-linear isomorphism

$$
\Sigma^{q} \approx D_{0}^{q} .
$$

This isomorphism

$$
\Sigma^{*} \approx D_{0}^{*}
$$

is not a homomorphism of sheaves of graded Lie algebras if $\Sigma^{*}$ has the graded Lie algebra structure described in $\S 5$, corresponding to the case that $\sigma \in \Sigma^{*}$ acts on $\Phi \in \mathcal{S}$ to give $\sigma \cdot \Phi$. Under (92) an element $\sigma \in \Sigma^{*}$ determines the derivation in $D_{0}^{*}$ given by

$$
\sigma * \Phi=\sigma \cdot \Phi-\Phi \pi \Im \sigma, \quad \Phi \in \mathcal{S} .
$$

In the case of tangential structure, therefore, a second structure of graded Lie algebra (over $R$ ) can be imposed on $\Sigma^{*}$, corresponding to the action (93). Equivalently, the new structure is induced from the graded Lie algebra structure of $D_{0}^{*}$ by the isomorphism (92). If we denote the new bracket product of $\sigma \in \Sigma^{q}, \tau \in \Sigma^{r}$ by $\{\sigma, \tau\} \in \Sigma^{q+r}$, then $\{\sigma, \tau\}$ is determined (uniquely, since ${ }_{0}^{1} S^{0} \mathrm{CS}$ ) by the condition

$$
\{\sigma, \tau\} * \Phi=\sigma *(\tau * \Phi)-(-1)^{a r} \tau *(\sigma * \Phi), \quad \Phi \in \delta,
$$


analogous to (39). An explicit computation of the right side of (94), using (93), (39), (25), and (57) shows that

$$
\{\sigma, \tau\}=[\sigma, \tau]-\left(\tau \pi J-(-1)^{q r} \sigma \pi \Im \tau\right), \quad \sigma \in \Sigma^{q}, \tau \in \Sigma^{r},
$$

with

(96) $J\{\sigma, \tau\}=-\left(\Im \tau \pi \Im \sigma-(-1)^{q r} \Im \sigma \pi J \tau\right)+(-1)^{r}[\Im \sigma, \pi \tau]-(-1)^{q r+q}[\Im \tau, \pi \sigma]$.

Clearly, the new graded Lie algebra structure on $\Sigma^{*}$ is not compatible with the exact sequence (46) of sheaves of graded $A^{0}$-modules; that is, $\Xi^{*}$ is not a graded ideal with respect to this structure, and no graded Lie algebra structure is induced on $B^{*}$. From (95) we see that the two graded Lie algebra structures on $\Sigma^{*}$ coincide on the subalgebra $L^{*}$ of torsionless operators defined by (89). Moreover, the graded submodule $\Lambda^{*}$, defined by (90), is a graded ideal of $\Sigma^{*}$ with respect to the new structure. In fact, it follows from (96) and (95) that

$$
J\{\lambda, \sigma\}=0, \quad \pi\{\lambda, \sigma\}=0, \quad \lambda \in \Lambda^{*}, \sigma \in \Sigma^{*},
$$

since $\Xi \lambda=0, \pi \lambda=0$ for $\lambda \in \Lambda^{*}$.

In the exact sequence

$$
0 \rightarrow \Lambda^{*} \rightarrow \Sigma^{*} \rightarrow Q^{*} \rightarrow 0
$$

of sheaves of graded Lie algebras (new structure), the quotient $Q^{*}$ is isomorphic to a graded Lie algebra of derivations of the exterior algebra $A^{*}={ }_{0}^{0} S^{*}$ on $M$, as has been pointed out by $\mathrm{D}$. C. Spencer. In fact, it follows from (93) and (24) that the compatible derivation of $A^{*}$ corresponding to $\sigma \in \Sigma^{*}$ is given by

$$
\sigma * \phi=d_{\pi \sigma} \phi-\phi \pi J \sigma, \quad \sigma \in \Sigma^{*}, \phi \in A^{*} ;
$$

in particular, the elements of $\Sigma^{*}$ which correspond to the zero derivation of $A^{*}$ are exactly the elements of $\Lambda^{*}$.

We shall verify below that, in the general case: $G=G L(n, R)$, the graded sheaf $Q^{*}$ corresponds to the graded sheaf of germs of all derivations of $A^{*}$ (except those of degree -1 , which are not compatible with tangential structure). In the case of a $G$-structure on $M$, the graded sheaf $Q^{*}$ determines a graded subsheaf of the sheaf of germs of all derivations of $A^{*}$, constituting those derivations of $A^{*}$ which are $a d m i s s i b l e$ under the given $G$-structure on $M$. The graded sheaf of germs of admissible derivations of $A^{*}$ has been studied by Kodaira and Spencer [4] in the case that the $G$-structure on $M$ is multifoliate structure.

Among the derivations of $A^{*}$, there is a distinguished (global) derivation, of degree 1 , corresponding to the exterior derivative $d$. The exterior derivative is an admissible derivation for a given $G$-structure on $M$ if and only if there exists a torsionless connection in the principal bundle of the tangent bundle, cor- 
responding to this $G$-structure, that is, for the principal bundle $P \rightarrow M$, with group $G$, of the tangent bundle of $M$, there is a connection form $\chi \in H^{0}\left(M, \Sigma^{1}\right)$ with $\pi \chi=\delta \in H^{0}\left(M, B^{1}\right)$ which satisfies

$$
\Im x=0 .
$$

In fact, if such a connection $\chi$ exists, then by (98)

$$
\chi * \phi=d \phi,
$$$$
\phi \in A^{*},
$$

since $d_{\delta}=d$. Conversely, if $d$ is an admissible derivation on all of $M$, then for a suitable open covering $\left\{U_{i}\right\}$ of $M$ (or any refinement of this covering) there exist local connection forms $\chi_{i} \in H^{0}\left(U_{i}, \Sigma^{1}\right)$ with $\pi \chi_{i}=\delta$ and $\Im \chi_{i}=0$. If $U_{i} \cap U_{k}$ $\neq \varnothing$, we have

$$
\chi_{i}-\chi_{k}=\lambda_{i k} \in H^{0}\left(U_{i} \cap U_{k}, \Lambda^{1}\right) \text {, }
$$

since $\pi \chi_{i}=\pi \chi_{k}, J \chi_{i}=J \chi_{k}(=0)$ on $U_{i} \cap U_{k}$. The sheaf $\Lambda^{1}$ is fine, since $M$ is paracompact and $\Lambda^{1}$ is a sheaf of germs of $A^{0}$-modules (since $\mathfrak{I}$ is $A^{0}$-linear on $\left.\Xi^{1}\right)$; therefore $H^{1}\left(M, \Lambda^{1}\right)=0$. Thus for the given covering, or a suitable refinement (denoted by the same symbols), we have

$$
\lambda_{i k}=\lambda_{k}-\lambda_{i}, \quad \lambda_{i} \in H^{0}\left(U_{i}, \Lambda^{1}\right), \lambda_{k} \in H^{0}\left(U_{k}, \Lambda^{1}\right),
$$

and

$$
\chi=\chi_{i}+\lambda_{i}=\chi_{k}+\lambda_{k}
$$

defines a torsionless connection for the principal bundle $P \rightarrow M$.

We remark that $J \chi$ as defined here coincides with the usual torsion of a connection. If $\chi$ is represented locally by $\pi \chi=\delta$ and an $(n \times n)$-matrix $\left(\omega_{\nu}^{\mu}\right)$ of 1 -forms, and the corresponding representation of $\mu \delta$ is given by 1 -forms $\omega^{\mu}, \mu=1, \cdots, n$, then (91) gives

$$
(\Im \chi)^{\mu}=d \omega^{\mu}-\omega_{\nu}^{\mu} \wedge \omega^{\nu}, \quad \quad \mu=1, \cdots, n .
$$

In the general case, $G=G L(n, R)$, or in the case that the $G$-structure is integrable, the local representation may be chosen so that $\omega^{\alpha}=d x^{\alpha}$, where $\left(x^{1}, \cdots, x^{n}\right)$ are local coordinates on $M$. If we set

$$
\omega_{\nu}^{\mu}=-\Gamma_{\nu \alpha}^{\mu} d x^{\alpha}
$$

then

$$
(\Im \chi)^{\mu}=\left(\Gamma_{\nu \alpha}^{\mu} d x^{\alpha}\right) \wedge d x^{\nu}, \quad \quad \mu=1, \cdots, n ;
$$

thus the condition $J \chi=0$ is expressed by

$$
\Gamma_{\nu \alpha}^{\mu}=\Gamma_{\alpha \nu}^{\mu}, \quad \alpha, \mu, \nu=1, \cdots, n .
$$


For the curvature form $R$, defined by (61), corresponding to a given connection form $\chi$, we have by (88)

$$
\mu \Im R=\chi \cdot \mu \Im \chi,
$$

which is the (first) Bianchi identity; in the case of a torsionless connection: $\Im \chi=0$, the formula (102) gives

$$
\Im R=0
$$

In a local representation, $R$ is given by $\pi R=0$ and an $(n \times n)$-matrix $\left(R_{\nu}^{\mu}\right)$ of 2 -forms

say; then, by (91),

$$
R_{\nu}^{\mu}=d \omega_{\nu}^{\mu}-\omega_{\rho}^{\mu} \wedge \omega_{\nu}^{p}=\sum_{\alpha<\beta} R_{\nu \alpha \beta}^{\mu} \omega^{\alpha} \wedge \omega^{\beta}
$$

$$
(J R)^{\mu}=-R_{\nu}^{\mu} \wedge \omega^{\nu}=-\left(\sum_{\alpha<\beta} R_{\nu \alpha \beta}^{\mu} \omega^{\alpha} \wedge \omega^{\beta}\right) \wedge \omega^{\nu},
$$

and (103) gives the usual formula

$$
R_{\nu \alpha \beta}^{\mu}+R_{\alpha \beta \nu}^{\mu}+R_{\beta \nu \alpha}^{\mu}=0, \quad \mu, \nu, \alpha, \beta=1, \cdots, n .
$$

In the case of tangential structure the exact sequence

$$
0 \rightarrow \Xi^{*} \stackrel{\iota}{\rightarrow} \Sigma^{*} \stackrel{\pi}{\rightarrow} B^{*} \rightarrow 0
$$

of sheaves of graded Lie algebras (over $R$ ) has a distinguished subsequence

$$
0 \rightarrow \Lambda^{*} \stackrel{\iota}{\rightarrow} L^{*} \stackrel{\pi}{\rightarrow} \Theta^{*} \rightarrow 0
$$

of sheaves of graded Lie algebras (over $R$ ) corresponding to the torsionless operators. (Since $\Lambda^{*}$ is a graded ideal in $L^{*}$, the quotient $\Theta^{*}$ is a graded subalgebra of the graded Lie algebra $B^{*}$.)

In all cases we have

$$
\Lambda^{0}=0, \quad L^{0} \approx \Theta^{0} .
$$

In fact, for $\Phi=\mu \delta$, the identity (57) becomes

(105) $(\sigma \cdot \mu \delta) \pi V-(-1)^{a s} \sigma \cdot \mu V=(\sigma \pi V) \cdot \mu \delta+(-1)^{q^{a}} \mu[V, \pi \sigma], \sigma \in \Sigma^{q}, V \in B^{s+1}$.

For $\sigma=\lambda \in \Lambda^{0}$, we have $\lambda \cdot \mu \delta=\lambda \pi V=\pi \lambda=0$, or

$$
\lambda \cdot \mu V=0
$$

$V \in B^{*}$,

which implies $\lambda=0$. For $\sigma \in L^{0},(105)$ reduces to

$$
\sigma \cdot \mu V=\mu[\pi \sigma, V] \text {, }
$$

$V \in B^{*}$.

Thus, for $V \in B^{*}$, the action of $\sigma \in L^{0}$ on $\mu V$ corresponds to the Lie derivative of 
$V$ with respect to $\pi \sigma \in \Theta^{0}$, since the Lie derivative of $V$ with respect to $\pi \sigma$ is expressed by the generalized Poisson bracket [2]. For $G=G L(n, R)$, we have $\Theta^{0}=B^{0}$, as will be seen below. Thus, for each $v \in B^{0}$, there is a unique $\sigma \in L^{0}$ with $\pi \sigma=v$; the right-invariant vector field on $P$ represented by this $\sigma$ is the vector field on $P$ "associated" with the vector field $v$ on $M$, in the terminology of Lichnerowicz [5]. For $G \subset G L(n, R)$, the elements of $\Theta^{0}$ are the (germs of) infinitesimal transformations corresponding to the pseudogroup determined by the $G$-structure on $M$ : they are precisely the elements for which the corresponding Lie derivation is an admissible derivation, relative to the given $G$-structure.

For $q>0$, it follows from (98) that $\Theta^{q}$ consists of those elements $V$ of $B^{q}$ for which the corresponding generalized Lie derivation $d_{V}$ on forms is admissible. In general, however, $\Lambda^{q} \neq 0$, and there is not a unique $\sigma \in L^{q}$ with $\pi \sigma=V$. In particular, there is a torsionless connection for the principal bundle of the tangent bundle if and only if $\delta \in H^{0}\left(M, \Theta^{1}\right)$.

The local study of the above sheaves and maps reduces to the consideration of the solutions of the system

$$
\sigma_{\nu}^{\mu} \wedge \omega^{\nu}=\psi^{\mu}, \quad \mu=1, \cdots, n,
$$

of equations for the $q$-forms $\sigma_{\nu}^{\mu}$ (where $\left\{\omega^{\mu}\right\}$ is a local basis (over $A^{0}$ ) for $A^{1}$ $={ }_{0}^{0} S^{1} \approx{ }_{1}^{0} S^{0}$ and where the $(q+1)$-forms $\psi^{\mu}$ are given) subject to the side condition, which is vacuous if $G=G L(n, R)$, that the matrix $\left(\sigma_{\nu}^{\mu}\right)$ be a $g$-valued $q$-form. The solutions of the homogeneous system $\left(\psi^{\mu}=0\right)$ give the local representations of the elements of $\Lambda^{q}$. Since $\left\{\omega^{\mu}\right\}$ is a basis, the local consideration also shows that $\Lambda^{0}=0$. An element $V$ in $B^{q}$ lies in $\Theta^{q}$ if and only if there is a solution of (107) for $\psi^{\mu}=d_{V} \omega^{\mu}$. In fact, the element $\sigma$ represented locally by $\pi \sigma=V$ and the $(n \times n)$-matrix $\left(\sigma_{p}^{\mu}\right)$ satisfying (107) then satisfies $J \sigma=0$ by (91); that is, $\sigma \in L^{q}$. A derivation $\phi \rightarrow d_{V} \phi+\phi \pi W$ of $A^{*}$, where $V \in B^{q}$, $W \in B^{q+1}$, is represented in the image $Q^{*}$ in (97) if and only if (107) can be solved when

$$
\psi^{\mu}=d_{V} \omega^{\mu}+W^{\mu}
$$

In fact, the corresponding $\sigma \in \Sigma^{q}$ then satisfies $\Xi \sigma=-W$ by (91), and $\sigma * \phi$ $=d_{V} \phi+\phi \pi W$.

In the case $G=G L(n, R)$, when the forms $\sigma_{v}^{\mu}$ need satisfy only (107), there are always solutions and we have $\Lambda^{q} \neq 0$ for $q>0, \Theta^{*}=B^{*}$, and $Q^{*}$ consists of all derivations of $A^{*}$ (except those of degree -1 ).

For $G \subset G L(n, R)$, the solutions $\sigma_{\nu}^{\mu}$ of $(107)$ are further restricted by the side condition that the matrix $\left(\sigma_{\nu}^{\mu}\right)$ define a $g$-valued $q$-form. The nature of this restriction depends on the choice of $G \subset G L(n, R)$, and different choices appear to require separate investigations.

For example, in the case $G=O(n)$, the side condition is 


$$
\sigma_{\nu}^{\mu}=-\sigma_{\mu}^{\nu}, \quad \quad \mu, \nu=1, \cdots, n .
$$

Thus $v \in B^{0}$ lies in $\Theta^{0}$ if and only if, in a local representation, the 1 -forms $d_{\nu} \omega^{\mu}=f_{\nu}^{\mu} \omega^{\nu}$, say, satisfy $f_{\nu}^{\mu}=-f_{\mu}^{\nu}$. We remark that this condition does not hold for arbitrary choices of a basis $\left\{\omega^{\mu}\right\}$ for 1-forms, but only for a basis which comes from a choice of coordinate function for the given principal bundle. In the case $G=O(n)$, such a basis will be one which is orthonormal relative to the Riemannian metric on $M$ corresponding to the given $O(n)$-structure on $M$. For $q=1$, it can be verified that the solutions of the homogeneous system (107) also satisfy $\sigma_{\nu}^{\mu}=\sigma_{\mu}^{\nu}$, so that we have

$$
\Lambda^{1}=0
$$

in the case $G=O(n)$. Again, for $q=1$, we have $\delta \in H^{0}\left(M, \Theta^{1}\right)$, since it is always possible to write

$$
d_{\delta} \omega^{\mu}=d \omega^{\mu}=\omega_{\nu}{ }^{\mu} \Lambda \omega^{\prime},
$$

where the 1 -forms $\omega_{\nu}^{\mu}$ satisfy $\omega_{\nu}^{\mu}=-\omega_{\mu}^{\nu}$. By (109) the solution $\sigma_{\nu}^{\mu}=\omega_{\nu}^{\mu}$ is the only solution of the equations

$$
\sigma_{\nu}{ }_{\nu} \wedge \omega^{\nu}=d \omega^{\mu}, \quad \mu=1, \cdots, n .
$$

That is, there is one and only one torsionless connection corresponding to a given $O(n)$-structure on $M$.

\section{REFERENCES}

1. M. F. Atiyah, Complex analytic connections in fibre bundles, Trans. Amer. Math. Soc. vol. 85 (1957) pp. 181-207.

2. A. Frölicher and A. Nijenhuis, Theory of vector-valued differential forms, I. Derivations in the graded ring of differential forms, Nederl. Akad. Wetensch. Proc. Ser. A. vol. 59 (1956) pp. 338-359.

3. S. Kobayashi, Theory of connections, Thesis, University of Washington, 1956.

4. K. Kodaira and D. C. Spencer, Multifoliate structures, Ann. of Math. vol. 74 (1961), to appear.

5. A. Lichnerowicz, Géométrie des groupes de transformations, Paris, Dunod, 1958.

Princeton University,

Princeton, New Jersey 